\title{
Certain Concepts of Vague Graphs With Applications to Medical Diagnosis
}

\author{
Zehui Shao ${ }^{1}$, Saeed Kosari ${ }^{1 *}$, Muhammad Shoaib ${ }^{2}$ and Hossein Rashmanlou ${ }^{3}$ \\ ${ }^{1}$ Institute of Computing Science and Technology, Guangzhou University, Guangzhou, China, ${ }^{2}$ Department of Mathematics, \\ University of the Punjab, New Campus, Lahore, Pakistan, ${ }^{3}$ Mazandaran Adib Institute of Higher Education, Sari, Iran
}

The purpose of this research study is to present and explore the key properties of some new operations on vague graphs, including rejection, maximal product, symmetric difference, and residue product. This article introduces the notions of degree of a vertex and total degree of a vertex in a vague graph. As well, this study outlines the specific conditions required for obtaining the degrees of vertices in vague graphs under the operations of maximal product, symmetric difference, and rejection. The article also discusses applications of vague sets in medical diagnosis.

Keywords: vague set, maximal product, rejection, symmetric difference, residue product, application

\section{OPEN ACCESS}

Edited by:

Muhammad Javaid,

University of Management and

Technology, Pakistan

Reviewed by:

Anouar Ben Mabrouk,

University of Kairouan, Tunisia Ndolane Sene

Cheikh Anta Diop University, Senegal

*Correspondence:

Saeed Kosar

saeedkosari38@yahoo.com

Specialty section

This article was submitted to Mathematical and Statistical Physics,

a section of the journal

Frontiers in Physics

Received: 17 May 2020

Accepted: 27 July 2020

Published: 11 November 2020

Citation:

Shao Z, Kosari S, Shoaib M and

Rashmanlou H (2020) Certain

Concepts of Vague Graphs With Applications to Medical Diagnosis.

Front. Phys. 8:357.

doi: 10.3389/fphy.2020.00357

\section{INTRODUCTION}

Graph theory is an extremely useful tool for solving combinatorial problems in a wide range of fields, including geometry, algebra, number theory, topology, operations research, biology, and social systems. Graph theory also has many applications of great scope, such as in networking, image capture, clustering, handling uncertainty, image segmentation, finding communities in networks, bioscience, information technology, operations research, and social science networks consisting of points connected by lines. In fact, graph theory studies connections between objects, such as vertices and edges and the various relations between them. Fuzzy graph theory is finding an increasing number of applications in modeling real-time systems, where the amount of information inherent in the system varies with different levels of precision. In 1965, Zadeh [1] first proposed the theory of fuzzy sets. The fuzzy graph, with the approximate reasoning, enables many combinatorial problems in fields, such as topology and algebra to be solved more easily. The concept of fuzzy graphs is discussed by Rosenfeld [2] as well as by Bhattacharya [3, 4]. Fuzzy graphs date back to the nineteenth century, and their use has grown tremendously in recent years [5, 6]. Gau and Buehrer [7] proposed the concept of vague set in 1993, which replaces the value of an element in a set with a subinterval of $[0,1]$. Specifically, a true-membership function $t_{v}(x)$ and a false-membership function $f_{v}(x)$ are used to describe the boundaries of the membership degree. Descriptions of realworld problems can be improved by using the theory of vague sets. Researchers have applied this theory to several real-world situations, such as decision-making and fuzzy control. The theory of vague sets is also helpful for fault diagnosis and knowledge discovery. Interval-valued fuzzy sets have a case vague set, which has been applied in different fields of mathematics. Ramakrishna [8] introduced the concept of vague graph and also studied related properties. Vague graphs have numerous applications in geometry and operations research and are also useful in many areas of computer science. Rashmanlou and Borzooei [9] studied new concepts relating to vague graphs, product vague graphs [10], regularity of vague graphs [11], and vague competition graphs [12]. Krishna and Lavanya [13] developed new concepts of coloring in vague graphs. Besides the 
membership degree, the non-membership degree has been introduced as well, which is presented by Atanassove [14] in an intuitionistic fuzzy set, a type of extension of a fuzzy set. Parvathi and Karunambigai [15] discussed intuitionistic fuzzy graphs. Devi et al. [16] presented new concepts regarding intuitionistic fuzzy labeling graphs.

In this study we outline and explore the key properties of some new operations on vague graphs, including rejection, maximal product, symmetric difference, and residue product. We introduce new notions, such as degree of a vertex and total degree of a vertex in a vague graph. We also outline specific conditions for obtaining the degrees of vertices in vague graphs under the operations of maximal product, symmetric difference, and rejection. Furthermore, we explore applications of vague sets in medical diagnosis.

\section{PRELIMINARIES}

In this section we introduce the key preliminary notions and definitions that are used in this study.

Definition 2.1 ([17]). A graph is an ordered pair $G=(V, E)$, where $V$ is the set of vertices of $G$ and $E$ is the set of all edges, arcs, or lines, which are two-element subsets of $V$ (that is, an edge is related to two vertices and the relation is represented as an unordered pair $\{m, n\}$ of those vertices).

Note that for an edge $\{m, n\}$, graph theorists usually use the somewhat shorter notation $m n$. Two vertices $m$ and $n$ in an undirected graph $G$ are said to be adjacent in $G$ if $m n$ is an edge of $G$. An edge whose endpoints are the same is called a loop. A graph without loops is called a simple graph.

Definition 2.2 ([7]). A vague set $M$ is a pair $\left(T_{M} ; F_{M}\right)$ of functions on a set $V$, where $T_{M}$ and $F_{M}$ are real-valued $V \rightarrow$ $[0,1]$ functions such that $T_{M}(m)+F_{M}(m)<1$ for all $m \in V$. The interval $\left[T_{M}(m), 1-F_{M}(m)\right]$ is known as the vague value of $m$ in $M$.

In this definition, for $m$ in $M, T_{M}(m)$ is the lower bound for the degree of membership and $F_{M}(m)$ is the lower bound for the negative of the degree of membership. Therefore, the degree of membership of $m \in M$ is given by the interval $\left[T_{M}(m), 1-\right.$ $\left.F_{M}(m)\right]$.

Definition 2.3 ([8]). Let $G=(V, E)$ be a crisp graph. A pair $\mathbf{G}=$ $(M, N)$ is called a vague graph defined on the crisp graph $G=$ $(V, E)$ if $M=\left(T_{M}, F_{M}\right)$ is a vague set on $V$ and $N=\left(T_{N}, F_{N}\right)$ is vague set on $E \subseteq V \times V$ such that $T_{N}(m n) \leq \min \left(T_{M}(m), T_{M}(n)\right)$ and $F_{N}(m n) \geq \max \left(F_{M}(m), F_{M}(n)\right)$ for each edge $m n$ in $E$.

Definition 2.4 ([9]). A vague graph $\mathbf{G}$ is said to be strong if $T_{N}(m n)=\min \left(T_{M}(m), T_{M}(n)\right)$ and $F_{N}(m n)=$ $\max \left(F_{M}(m), F_{M}(n)\right)$ for all $m, n \in V$.

Definition 2.5 ([9]). A vague graph $\mathbf{G}$ is said to be complete if $T_{N}(m n)=\min \left(T_{M}(m), T_{M}(n)\right)$ and $F_{N}(m n)=\max \left(F_{M}(m), F_{M}(n)\right)$ for all $m n \in E$.

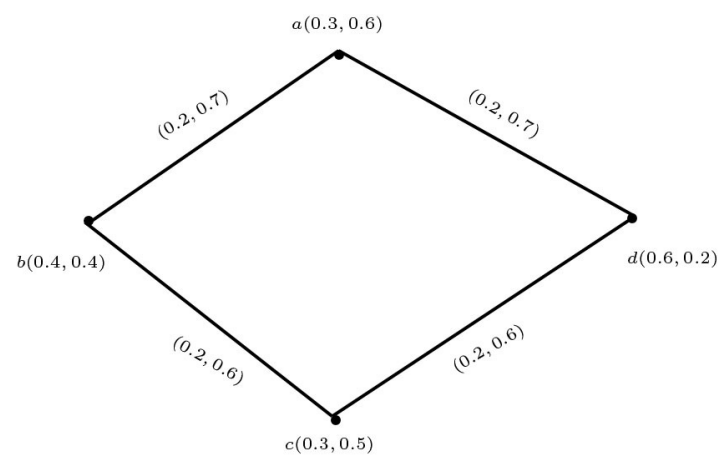

FIGURE 1 | The vague graph $\mathbf{G}$ in Example 2.7.

Definition 2.6 ([11]). A vague graph $\mathbf{G}$ is said to be connected if $T_{N}^{\infty}\left(m_{i} m_{j}\right)>0$ and $F_{N}^{\infty}\left(m_{i} m_{j}\right)<1$ for all $m_{i}, m_{j} \in V$. Also, we have

$$
\begin{array}{r}
T_{N}^{\infty}(m n)=\sup \left\{T_{N}\left(m n_{1}\right) \wedge T_{N}\left(n_{1} n_{2}\right) \wedge T_{N}\left(n_{2} n_{3}\right) \wedge \ldots\right. \\
\left.\wedge T_{N}\left(n_{k-1} n\right) \mid m, n_{1}, n_{2}, \ldots, n_{k-1}, n \in V\right\}
\end{array}
$$

and

$$
\begin{array}{r}
F_{N}^{\infty}(m n)=\inf \left\{F_{N}\left(m n_{1}\right) \vee F_{N}\left(n_{1} n_{2}\right) \vee F_{N}\left(n_{2} n_{3}\right) \vee \ldots\right. \\
\\
\left.\vee F_{N}\left(n_{k-1} n\right) \mid m, n_{1}, n_{2}, \ldots, n_{k-1}, n \in V\right\} .
\end{array}
$$

Example 2.7. Consider a vague graph $\mathbf{G}$ such that $V=\{a, b, c\}$, $E=\{a b, b c, c d, a d\}, M=\left\langle\left(\frac{a}{0.3}, \frac{b}{0.4}, \frac{c}{0.3}, \frac{d}{0.6}\right),\left(\frac{a}{0.6}, \frac{b}{0.4}, \frac{c}{0.5}, \frac{a}{0.2}\right)\right\rangle$, and $N=\left\langle\left(\frac{a b}{0.2}, \frac{b c}{0.2}, \frac{c d}{0.2}, \frac{a d}{0.2}\right),\left(\frac{a b}{0.7}, \frac{b c}{0.6}, \frac{c d}{0.6}, \frac{a d}{0.7}\right)\right\rangle$.

By routine computations, it is easy to show that $\mathbf{G}$ is a vague graph (Figure 1).

\section{OPERATIONS ON VAGUE GRAPHS}

In this section we define four new kinds of operations on vague graphs: the maximal product, residue product, rejection, and symmetric difference. We show that the maximal product, residue product, or rejection of two vague graphs is again a vague graph.

Definition 3.1. The maximal product $\mathbf{G}_{1} * \mathbf{G}_{2}=\left(M_{1} * M_{2}, N_{1} *\right.$ $\left.N_{2}\right)$ of two vague graphs $\mathbf{G}_{1}=\left(M_{1}, N_{1}\right)$ and $\mathbf{G}_{2}=\left(M_{2}, N_{2}\right)$ is defined by

$$
\begin{array}{r}
\left(T_{M_{1}} * T_{M_{2}}\right)\left(\left(m_{1}, m_{2}\right)\right)=\max \left\{T_{M_{1}}\left(m_{1}\right), T_{M_{2}}\left(m_{2}\right)\right\}, \\
\left(F_{M_{1}} * F_{M_{2}}\right)\left(\left(m_{1}, m_{2}\right)\right)=\min \left\{F_{M_{1}}\left(m_{1}\right), F_{M_{2}}\left(m_{2}\right)\right\} \\
\forall\left(m_{1}, m_{2}\right) \in\left(V_{1} \times V_{2}\right) ;
\end{array}
$$

(ii) $\left(T_{M_{1}} * T_{M_{2}}\right)\left(\left(m, m_{2}\right)\left(m, n_{2}\right)\right)=\max \left\{T_{M_{1}}(m), T_{N_{2}}\left(m_{2} n_{2}\right)\right\}$, $\left(F_{M_{1}} * F_{M_{2}}\right)\left(\left(m, m_{2}\right)\left(m, n_{2}\right)\right)=\min \left\{F_{M_{1}}(m), F_{N_{2}}\left(m_{2} n_{2}\right)\right\}$ $\forall m \in V_{1}$ and $m_{2} n_{2} \in E_{2}$;

(iii) $\left(T_{M_{1}} * T_{M_{2}}\right)\left(\left(m_{1}, z\right)\left(n_{1}, z\right)\right)=\max \left\{T_{N_{1}}\left(m_{1} n_{1}\right), T_{M_{2}}(z)\right\}$, $\left(F_{M_{1}} * F_{M_{2}}\right)\left(\left(m_{1}, z\right)\left(n_{1}, z\right)\right)=\min \left\{F_{N_{1}}\left(m_{1} n_{1}\right), F_{M_{2}}(z)\right\}$ $\forall z \in V_{2}$ and $m_{1} n_{1} \in E_{1}$. 
Example 3.2. Consider the two vague graphs $\mathbf{G}_{1}$ and $\mathbf{G}_{2}$ shown in Figures 2, 3. Their maximal product $\mathbf{G}_{1} * \mathbf{G}_{2}$ is shown in Figure 4.

For the vertex $(a, d)$, we find the membership and nonmembership values as follows:

$$
\begin{aligned}
\left(T_{M_{1}} * T_{M_{2}}\right)((a, d)) & =\max \left\{T_{M_{1}}(a), T_{M_{2}}(d)\right\} \\
& =\max \{0.4,0.1\}=0.4 \\
\left(F_{M_{1}} * F_{M_{2}}\right)((a, d))= & \min \left\{F_{M_{1}}(a), F_{M_{2}}(d)\right\} \\
= & \min \{0.5,0.3\}=0.3 \\
& \text { for } a \in V_{1} \text { and } d \in V_{2} .
\end{aligned}
$$

For the edge $(a, d)(a, e)$, we find the following membership and non-membership values:

$$
\begin{aligned}
\left(T_{M_{1}} * T_{M_{2}}\right)((a, d)(a, e))= & \max \left\{T_{M_{1}}(a), T_{N_{2}}(d e)\right\} \\
= & \max \{0.4,0.1\}=0.4, \\
\left(F_{M_{1}} * F_{M_{2}}\right)((a, d)(a, e))= & \min \left\{F_{M_{1}}(a), F_{N_{2}}(d e)\right\} \\
= & \min \{0.5,0.6\}=0.5, \\
& \text { for } a \in V_{1} \text { and } d e \in E_{2} .
\end{aligned}
$$

Now, for edge $(a, g)(b, g)$ we have

$$
\begin{aligned}
\left(T_{M_{1}} * T_{M_{2}}\right)((a, g)(b, g))= & \max \left\{T_{N_{1}}(a b), T_{M_{2}}(g)\right\} \\
= & \max \{0.2,0.3\}=0.3, \\
\left(F_{M_{1}} * F_{M_{2}}\right)((a, g)(b, g))= & \min \left\{F_{N_{1}}(a b), F_{M_{2}}(g)\right\} \\
= & \min \{0.7,0.4\}=0.4, \\
& \text { for } g \in V_{2} \text { and } a b \in E_{1} .
\end{aligned}
$$

Similarly, we can find the membership and non-membership values for all the remaining vertices and edges.

Proposition 3.3. The maximal product of two vague graphs $\mathbf{G}_{1}$ and $\mathbf{G}_{2}$ is a vague graph.

Proof: Let $\mathbf{G}_{1}=\left(M_{1}, N_{1}\right)$ and $\mathbf{G}_{2}=\left(M_{2}, N_{2}\right)$ be two vague graphs on crisp graphs $G_{1}=\left(V_{1}, E_{1}\right)$ and $G_{2}=\left(V_{2}, E_{2}\right)$, respectively, and let $\left(\left(m_{1}, m_{2}\right)\left(n_{1}, n_{2}\right)\right) \in E_{1} \times E_{2}$. Then by Definition 3.1 we have two cases:

(i) If $m_{1}=n_{1}=m$, then

$$
\begin{aligned}
& \left(T_{N_{1}} * T_{N_{2}}\right)\left(\left(m, m_{2}\right)\left(m, n_{2}\right)\right)=\max \left\{T_{M_{1}}(m), T_{N_{2}}\left(m_{2} n_{2}\right)\right\} \\
& \leq \max \left\{T_{M_{1}}(m), \min \left\{T_{M_{2}}\left(m_{2}\right), T_{M_{2}}\left(n_{2}\right)\right\}\right\} \\
& =\min \left\{\operatorname { m a x } \left\{\left\{T_{M_{1}}(m), T_{M_{2}}\left(m_{2}\right)\right\}, \max \left\{\left\{T_{M_{1}}(m), T_{M_{2}}\left(n_{2}\right)\right\}\right\}\right.\right. \\
& =\min \left\{\left(T_{M_{1}} * T_{M_{2}}\right)\left(m, m_{2}\right),\left(T_{M_{1}} * T_{M_{2}}\right)\left(m, n_{2}\right)\right\}, \\
& \left(F_{N_{1}} * F_{N_{2}}\right)\left(\left(m, m_{2}\right)\left(m, n_{2}\right)\right)=\min \left\{F_{M_{1}}(m), F_{N_{2}}\left(m_{2} n_{2}\right)\right\} \\
& \geq \min \left\{F_{M_{1}}(m), \max \left\{F_{M_{2}}\left(m_{2}\right), F_{M_{2}}\left(n_{2}\right)\right\}\right\} \\
& =\max \left\{\operatorname { m i n } \left\{\left\{F_{M_{1}}(m), F_{M_{2}}\left(m_{2}\right)\right\}, \min \left\{\left\{F_{M_{1}}(m), F_{M_{2}}\left(n_{2}\right)\right\}\right\}\right.\right. \\
& =\max \left\{\left(F_{M_{1}} * F_{M_{2}}\right)\left(m, m_{2}\right),\left(F_{M_{1}} * F_{M_{2}}\right)\left(m, n_{2}\right)\right\} .
\end{aligned}
$$

(ii) If $m_{2}=n_{2}=z$, then

$$
\begin{aligned}
& \left(T_{N_{1}} * T_{N_{2}}\right)\left(\left(m_{1}, z\right)\left(n_{1}, z\right)\right)=\max \left\{T_{N_{1}}\left(m_{1} n_{1}\right), T_{M_{2}}(z)\right\} \\
& \leq \max \left\{\min \left\{T_{N_{1}}\left(m_{1} n_{1}\right), T_{M_{2}}(z)\right\}\right. \\
& =\min \left\{\operatorname { m a x } \left\{\left\{T_{N_{1}}\left(m_{1}\right), T_{M_{2}}(z)\right\}, \max \left\{\left\{T_{M_{1}}\left(n_{1}\right), T_{M_{2}}(z)\right\}\right\}\right.\right. \\
& =\min \left\{\left(T_{M_{1}} * T_{M_{2}}\right)\left(m_{1}, z\right),\left(T_{M_{1}} * T_{M_{2}}\right)\left(n_{1}, z\right)\right\}, \\
& \left(F_{N_{1}} * F_{N_{2}}\right)\left(\left(m_{1}, z\right)\left(n_{1}, z\right)\right)=\min \left\{F_{N_{1}}\left(m_{1} n_{1}\right), F_{M_{2}}(z)\right\} \\
& \geq \min \left\{\max \left\{F_{N_{1}}\left(m_{1} n_{1}\right), F_{M_{2}}(z)\right\}\right. \\
& =\max \left\{\operatorname { m i n } \left\{\left\{F_{M_{1}}\left(m_{1}\right), F_{M_{2}}(z)\right\}, \min \left\{\left\{F_{M_{1}}\left(n_{1}\right), F_{M_{2}}(z)\right\}\right\}\right.\right. \\
& =\max \left\{\left(F_{M_{1}} * F_{M_{2}}\right)\left(m_{1}, z\right),\left(F_{M_{1}} * F_{M_{2}}\right)\left(n_{1}, z\right)\right\} .
\end{aligned}
$$

Therefore, $\mathbf{G}_{1} * \mathbf{G}_{2}$ is a vague graph.

Theorem 3.4. The maximal product of two strong vague graphs $\mathbf{G}_{1}$ and $\mathbf{G}_{2}$ is a strong vague graph.

Proof: Let $\mathbf{G}_{1}=\left(M_{1}, N_{1}\right)$ and $\mathbf{G}_{2}=\left(M_{2}, N_{2}\right)$ be two strong vague graphs on crisp graphs $G_{1}=\left(V_{1}, E_{1}\right)$ and $G_{2}=\left(V_{2}, E_{2}\right)$, respectively, and let $\left(\left(m_{1}, m_{2}\right)\left(n_{1}, n_{2}\right)\right) \in E_{1} \times E_{2}$. Then, by Proposition $3.3, \mathbf{G}_{1} * \mathbf{G}_{2}$ is a vague graph. Now we have two cases:

(i) If $m_{1}=n_{1}=m$, then

$$
\begin{aligned}
& \left(T_{N_{1}} * T_{N_{2}}\right)\left(\left(m, m_{2}\right)\left(m, n_{2}\right)\right)=\max \left\{T_{M_{1}}(m), T_{N_{2}}\left(m_{2} n_{2}\right)\right\} \\
& =\max \left\{T_{M_{1}}(m), \min \left\{T_{M_{2}}\left(m_{2}\right), T_{M_{2}}\left(n_{2}\right)\right\}\right\} \\
& =\min \left\{\operatorname { m a x } \left\{\left\{T_{M_{1}}(m), T_{M_{2}}\left(m_{2}\right)\right\}, \max \left\{\left\{T_{M_{1}}(m), T_{M_{2}}\left(n_{2}\right)\right\}\right\}\right.\right. \\
& =\min \left\{\left(T_{M_{1}} * T_{M_{2}}\right)\left(m, m_{2}\right),\left(T_{M_{1}} * T_{M_{2}}\right)\left(m, n_{2}\right)\right\} \\
& \left(F_{N_{1}} * F_{N_{2}}\right)\left(\left(m, m_{2}\right)\left(m, n_{2}\right)\right)=\min \left\{F_{M_{1}}(m), F_{N_{2}}\left(m_{2} n_{2}\right)\right\} \\
& =\min \left\{F_{M_{1}}(m), \max \left\{F_{M_{2}}\left(m_{2}\right), F_{M_{2}}\left(n_{2}\right)\right\}\right\} \\
& =\max \left\{\operatorname { m i n } \left\{\left\{F_{M_{1}}(m), F_{M_{2}}\left(m_{2}\right)\right\}, \min \left\{\left\{F_{M_{1}}(m), F_{M_{2}}\left(n_{2}\right)\right\}\right\}\right.\right. \\
& =\max \left\{\left(F_{M_{1}} * F_{M_{2}}\right)\left(m, m_{2}\right),\left(F_{M_{1}} * F_{M_{2}}\right)\left(m, n_{2}\right)\right\}
\end{aligned}
$$

(ii) If $m_{2}=n_{2}=z$, then

$$
\begin{aligned}
& \left(T_{N_{1}} * T_{N_{2}}\right)\left(\left(m_{1}, z\right)\left(n_{1}, z\right)\right)=\max \left\{T_{N_{1}}\left(m_{1} n_{1}\right), T_{M_{2}}(z)\right\} \\
& =\max \left\{\min \left\{T_{N_{1}}\left(m_{1} n_{1}\right), T_{M_{2}}(z)\right\}\right. \\
& =\min \left\{\operatorname { m a x } \left\{\left\{T_{N_{1}}\left(m_{1}\right), T_{M_{2}}(z)\right\}, \max \left\{\left\{T_{M_{1}}\left(n_{1}\right), T_{M_{2}}(z)\right\}\right\}\right.\right. \\
& =\min \left\{\left(T_{M_{1}} * T_{M_{2}}\right)\left(m_{1}, z\right),\left(T_{M_{1}} * T_{M_{2}}\right)\left(n_{1}, z\right)\right\}, \\
& \left(F_{N_{1}} * F_{N_{2}}\right)\left(\left(m_{1}, z\right)\left(n_{1}, z\right)\right)=\min \left\{F_{N_{1}}\left(m_{1} n_{1}\right), F_{M_{2}}(z)\right\} \\
& =\min \left\{\max \left\{F_{N_{1}}\left(m_{1} n_{1}\right), F_{M_{2}}(z)\right\}\right. \\
& =\max \left\{\operatorname { m i n } \left\{\left\{F_{M_{1}}\left(m_{1}\right), F_{M_{2}}(z)\right\}, \min \left\{\left\{F_{M_{1}}\left(n_{1}\right), F_{M_{2}}(z)\right\}\right\}\right.\right. \\
& =\max \left\{\left(F_{M_{1}} * F_{M_{2}}\right)\left(m_{1}, z\right),\left(F_{M_{1}} * F_{M_{2}}\right)\left(n_{1}, z\right)\right\} .
\end{aligned}
$$

Therefore, $\mathbf{G}_{1} * \mathbf{G}_{2}$ is a strong vague graph.

Example 3.5. Consider the strong vague graphs $\mathbf{G}_{1}$ and $\mathbf{G}_{2}$ as in Figure 5.

It is easy to see that $\mathbf{G}_{1} * \mathbf{G}_{2}$ is a strong vague graph too.

Remark 3.1. If the maximal product of two vague graphs $\mathbf{G}_{1}=$ $\left(M_{1}, N_{1}\right)$ and $\mathbf{G}_{2}=\left(M_{2}, N_{2}\right)$ is a strong vague graph, $\mathbf{G}_{1}$ and $\mathbf{G}_{2}$ need not be strong in general. 


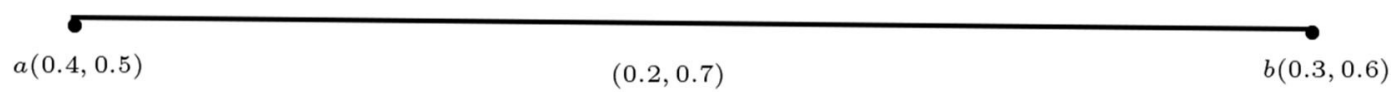

FIGURE 2 | $\mathbf{G}_{1}$

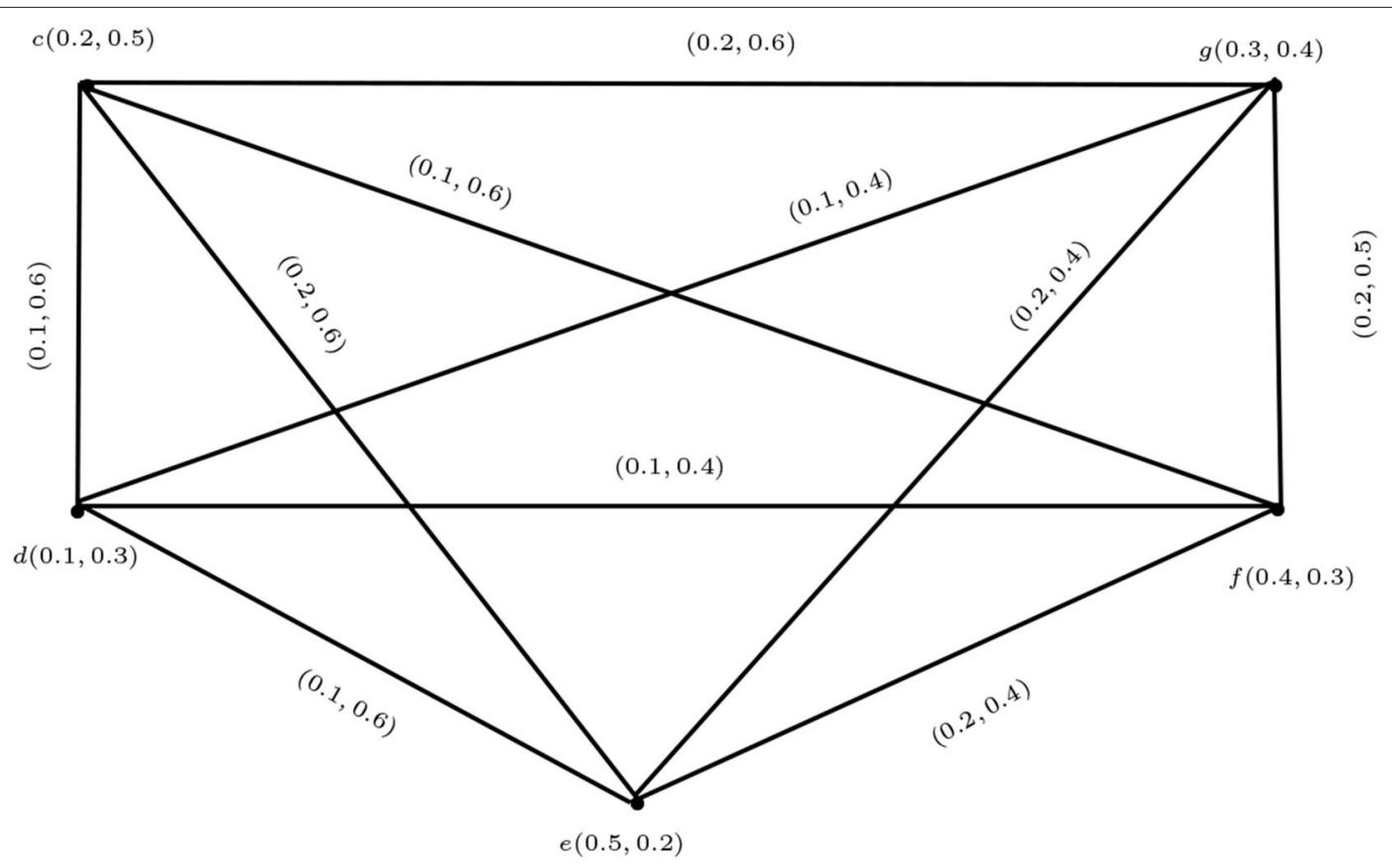

FIGURE 3 | $\mathbf{G}_{2}$

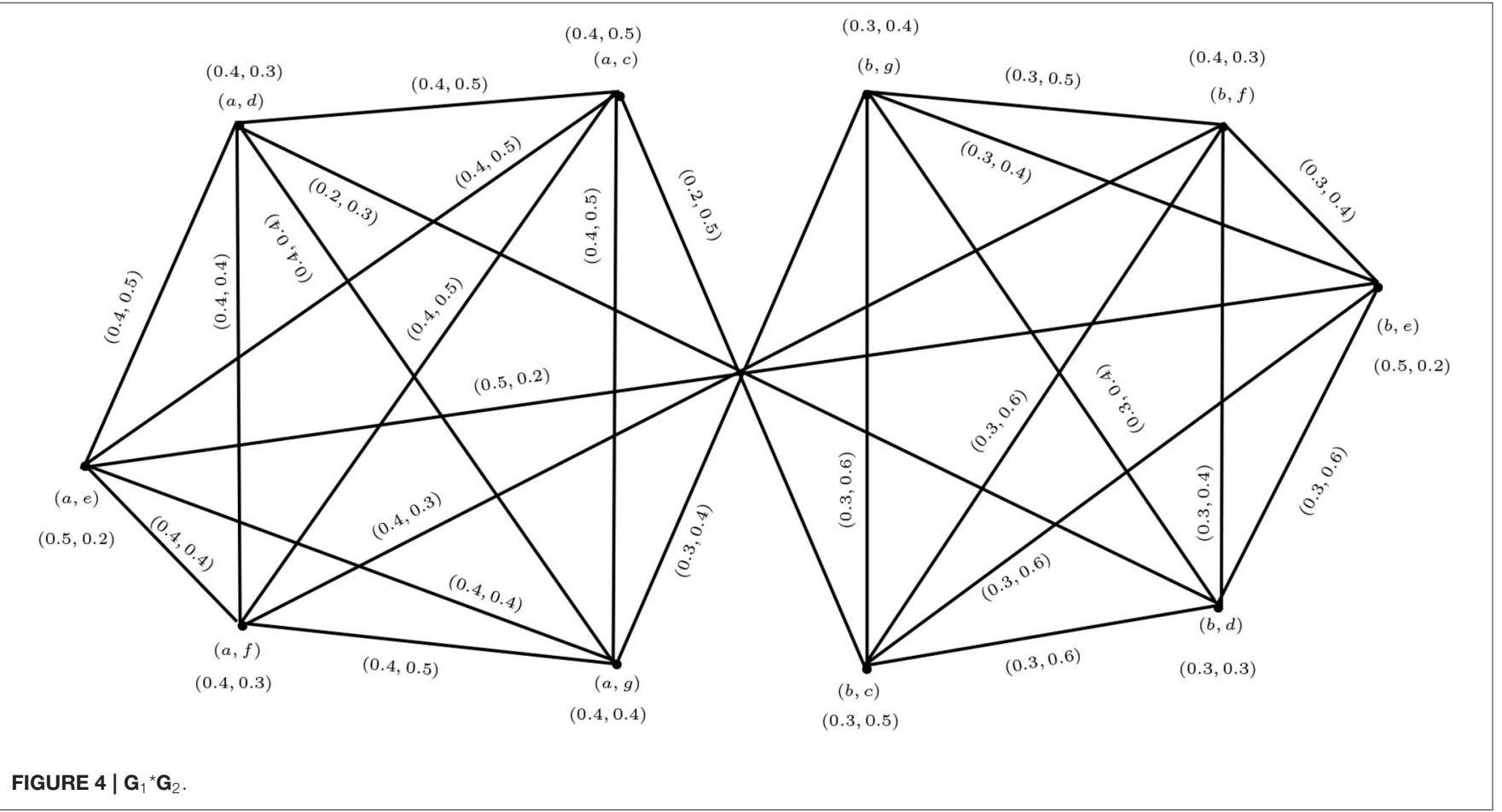




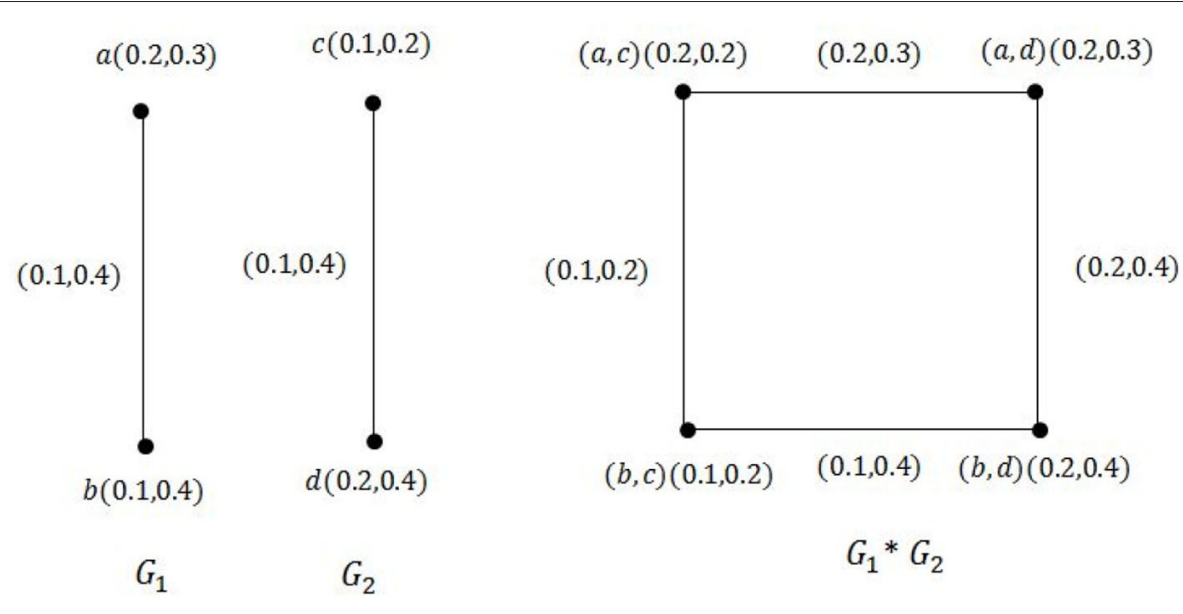

FIGURE 5 | Vague graphs $\mathbf{G}_{1}, \mathbf{G}_{2}$, and $\mathbf{G}_{1}{ }^{\star} \mathbf{G}_{2}$.

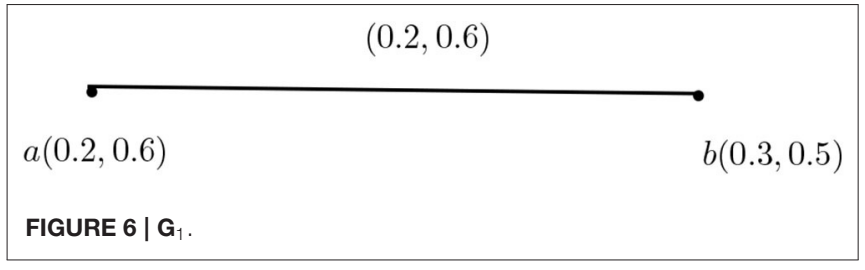

\begin{tabular}{|l|l|}
\hline & $(0.1,0.7)$ \\
$c(0.2,0.6)$ & $d(0.2,0.7)$ \\
FIGURE 7 | $\mathbf{G}_{2}$. & \\
\hline
\end{tabular}

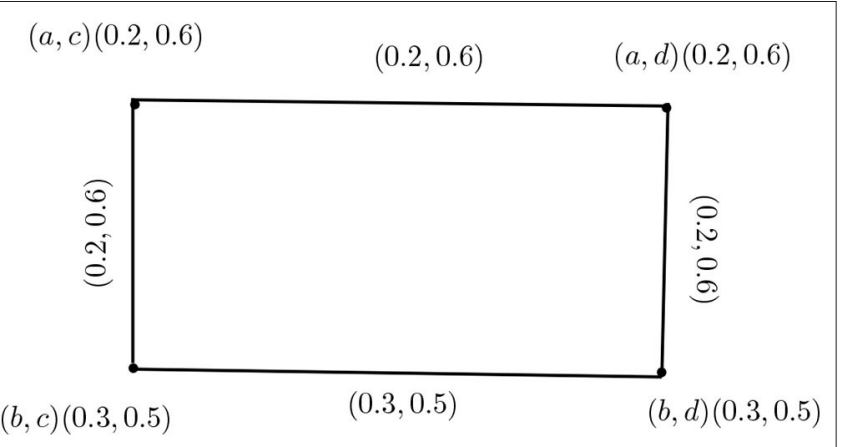

FIGURE $8 \mid \mathbf{G}_{1} * \mathbf{G}_{2}$.

Example 3.6. Consider the vague graphs $\mathbf{G}_{1}$ and $\mathbf{G}_{2}$ as in Figures 6 , 7. The maximal product of $\mathbf{G}_{1}$ and $\mathbf{G}_{2}$ is $\mathbf{G}_{1} * \mathbf{G}_{2}$ shown in Figure 8.

We can see that $\mathbf{G}_{1}$ and $\mathbf{G}_{1} * \mathbf{G}_{2}$ are strong vague graphs, but $\mathbf{G}_{2}$ is not strong: since $T_{N_{2}}\left(m_{2}, n_{2}\right)=0.1$ but $\min \left\{T_{M_{2}}\left(m_{2}\right), T_{M_{2}}\left(n_{2}\right\}=\min \{0.2,0.2\}=0.2\right.$, we have $T_{N_{2}}\left(m_{2}, n_{2}\right) \neq \min \left\{T_{M_{2}}\left(m_{2}\right), T_{M_{2}}\left(n_{2}\right\}\right.$.

Theorem 3.7. The maximal product of two connected vague graphs is a connected vague graph.

Proof: Let $\mathbf{G}_{1}=\left(M_{1}, N_{1}\right)$ and $\mathbf{G}_{2}=\left(M_{2}, N_{2}\right)$ be two connected vague graphs on crisp graphs $G_{1}=\left(V_{1}, E_{1}\right)$ and $G_{2}=$ $\left(V_{2}, E_{2}\right)$, respectively, where $V_{1}=\left\{m_{1}, m_{2}, \ldots, m_{k}\right\}$ and $V_{2}=$ $\left\{n_{1}, n_{2}, \ldots, n_{s}\right\}$. Then $T_{N_{1}}^{\infty}\left(m_{i} m_{j}\right)>0$ for all $m_{i}, m_{j} \in V_{1}$ and $T_{N_{2}}^{\infty}\left(n_{i} n_{j}\right)>0$ for all $n_{i}, n_{j} \in V_{2}$ (or $F_{N_{1}}^{\infty}\left(m_{i} m_{j}\right)<1$ for all $m_{i}, m_{j} \in V_{1}$ and $F_{N_{2}}^{\infty}\left(n_{i} n_{j}\right)<1$ for all $\left.n_{i}, n_{j} \in V_{2}\right)$. The maximal product of $\mathbf{G}_{1}=\left(M_{1}, N_{1}\right)$ and $\mathbf{G}_{2}=\left(M_{2}, N_{2}\right)$ can be taken as $\mathbf{G}=(M, N)$. Now, consider the $k$ subgraphs of $\mathbf{G}$ with the vertex set $\left\{\left(m_{i}, n_{1}\right),\left(m_{i}, n_{2}\right), \ldots,\left(m_{i}, n_{s}\right)\right\}$ for $i=1,2, \ldots, k$. Each of these subgraphs of $\mathbf{G}$ is connected, since the $m_{i}$ 's are the same and $\mathbf{G}_{2}$ is connected, so that each $n_{i}$ is adjacent to at least one of the vertices in $V_{2}$. Also, since $\mathbf{G}_{1}$ is connected, each $x_{i}$ is adjacent to at least one of the vertices in $V_{1}$.

Hence, there exists at least one edge between any pair of the above $k$ subgraphs. Thus, we have $T_{N}^{\infty}\left(\left(m_{i}, n_{j}\right)\left(m_{m}, n_{n}\right)\right)>0$ (or $\left.F_{N}^{\infty}\left(\left(m_{i}, n_{j}\right)\left(m_{m}, n_{n}\right)\right)<1\right)$ for all $\left(\left(m_{i}, n_{j}\right)\left(m_{m}, n_{n}\right)\right) \in E$. Therefore, $G$ is a connected vague graph.

Remark 3.2. The maximal product of two complete vague graphs is not a complete vague graph in general. This is because we do not include the case where $\left(m_{1}, m_{2}\right) \in E_{1}$ and $\left(n_{1}, n_{2}\right) \in E_{2}$ in the definition of the maximal product of two vague graphs.

Remark 3.3. The maximal product of two complete vague graphs is a strong vague graph.

Example 3.8. Consider the complete vague graphs $\mathbf{G}_{1}$ and $\mathbf{G}_{2}$ in Figure 5. A simple calculation yields that $\mathbf{G}_{1} * \mathbf{G}_{2}$ is a strong vague graph. 


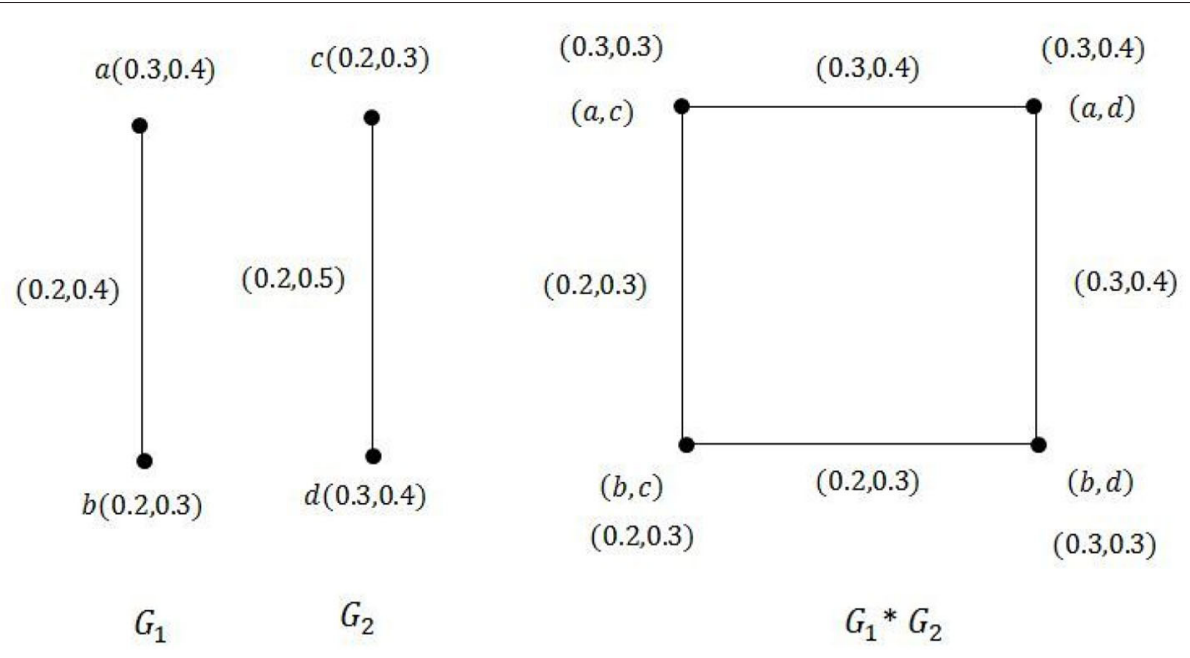

FIGURE 9 | Vague graphs $\mathbf{G}_{1}, \mathbf{G}_{2}$, and $\mathbf{G}_{1} * \mathbf{G}_{2}$.

Definition 3.9. Let $\mathbf{G}_{1}=\left(M_{1}, N_{1}\right)$ and $\mathbf{G}_{2}=\left(M_{2}, N_{2}\right)$ be two vague graphs. For any vertex $\left(m_{1}, m_{2}\right) \in V_{1} \times V_{2}$ we define

$$
\begin{array}{r}
\left(d_{T}\right)_{\mathbf{G}_{1} * \mathbf{G}_{2}}\left(m_{1}, m_{2}\right)=\sum_{\left(m_{1}, m_{2}\right)\left(n_{1}, n_{2}\right) \in E_{1} \times E_{2}}\left(T_{N_{1}} * T_{N_{2}}\right)\left(\left(m_{1}, m_{2}\right)\left(n_{1}, n_{2}\right)\right) \\
=\sum_{m_{1}=n_{1}, m_{2} n_{2} \in E_{2}} \max \left\{T_{M_{1}}\left(m_{1}\right), T_{N_{2}}\left(m_{2} n_{2}\right)\right\} \\
+\sum_{m_{1} n_{1} \in E_{1}, m_{2}=n_{2}} \max \left\{T_{N_{1}}\left(m_{1} n_{1}\right), T_{M_{2}}\left(m_{2}\right)\right\}, \\
\left(d_{F}\right)_{\mathbf{G}_{1} * \mathbf{G}_{2}}\left(m_{1}, m_{2}\right) \\
=\sum_{\left(m_{1}, m_{2}\right)\left(n_{1}, n_{2}\right) \in E_{1} \times E_{2}}\left(F_{N_{1}} * F_{N_{2}}\right)\left(\left(m_{1}, m_{2}\right)\left(n_{1}, n_{2}\right)\right) \\
=\sum_{m_{1}=n_{1}, m_{2} n_{2} \in E_{2}} \min \left\{F_{M_{1}}\left(m_{1}\right), F_{N_{2}}\left(m_{2} n_{2}\right)\right\} \\
+\sum_{m_{1} n_{1} \in E_{1}, m_{2}=n_{2}} \min \left\{F_{N_{1}}\left(m_{1} n_{1}\right), F_{M_{2}}\left(m_{2}\right)\right\} .
\end{array}
$$

Theorem 3.10. Let $\mathbf{G}_{1}=\left(M_{1}, N_{1}\right)$ and $\mathbf{G}_{2}=\left(M_{2}, N_{2}\right)$ be two vague graphs. If $T_{M_{1}} \geq T_{N_{2}}, F_{M_{1}} \leq F_{N_{2}}$, $T_{M_{2}} \geq T_{N_{1}}$, and $F_{M_{2}} \leq F_{N_{1}}$, then $\left(d_{T}\right)_{\mathbf{G}_{1} * \mathbf{G}_{2}}\left(m_{1}, m_{2}\right)=$ $(d)_{G_{2}}\left(m_{2}\right) T_{M_{1}}\left(m_{1}\right)+(d)_{G_{1}}\left(m_{1}\right) T_{M_{2}}\left(m_{2}\right) \quad$ and $\left(d_{F}\right)_{\mathbf{G}_{1} * \mathbf{G}_{2}}\left(m_{1}, m_{2}\right)=(d)_{G_{2}}\left(m_{2}\right) F_{M_{1}}\left(m_{1}\right)+(d)_{G_{1}}\left(m_{1}\right) F_{M_{2}}\left(m_{2}\right)$.

Proof: From the definition of a vertex in the cartesian product, we have

$$
\begin{aligned}
\left(d_{T}\right)_{\mathbf{G}_{1} * \mathbf{G}_{2}}\left(m_{1}, m_{2}\right) & =\sum_{\left(m_{1}, m_{2}\right)\left(n_{1}, n_{2}\right) \in E_{1} \times E_{2}}\left(T_{N_{1}} * T_{N_{2}}\right)\left(\left(m_{1}, m_{2}\right)\left(n_{1}, n_{2}\right)\right) \\
& =\sum_{m_{1}=n_{1}, m_{2} n_{2} \in E_{2}} \max \left\{T_{M_{1}}\left(m_{1}\right), T_{N_{2}}\left(m_{2} n_{2}\right)\right\} \\
& +\sum_{m_{1} n_{1} \in E_{1}, m_{2}=n_{2}} \max \left\{T_{N_{1}}\left(m_{1} n_{1}\right), T_{M_{2}}\left(m_{2}\right)\right\} \\
& =\sum_{m_{2} n_{2} \in E_{2}, m_{1}=n_{1}} T_{N_{2}}\left(m_{2} n_{2}\right)+\sum_{m_{1} n_{1} \in E_{1}, m_{2}=n_{2}} T_{N_{1}}\left(m_{1} n_{1}\right) \\
& =(d)_{G_{2}}\left(m_{2}\right) T_{M_{1}}\left(m_{1}\right)+(d)_{G_{1}}\left(m_{1}\right) T_{M_{2}}\left(m_{2}\right),
\end{aligned}
$$

$$
\begin{aligned}
\left(d_{F}\right)_{\mathbf{G}_{1} * \mathbf{G}_{2}}\left(m_{1}, m_{2}\right) & =\sum_{\left(m_{1}, m_{2}\right)\left(n_{1}, n_{2}\right) \in E_{1} \times E_{2}}\left(F_{N_{1}} * F_{N_{2}}\right)\left(\left(m_{1}, m_{2}\right)\left(n_{1}, n_{2}\right)\right) \\
& =\sum_{m_{1}=n_{1}, m_{2} n_{2} \in E_{2}} \min \left\{F_{M_{1}}\left(m_{1}\right), F_{N_{2}}\left(m_{2} n_{2}\right)\right\} \\
& +\sum_{m_{1} n_{1} \in E_{1}, m_{2}=n_{2}} \min \left\{F_{N_{1}}\left(m_{1} n_{1}\right), F_{M_{2}}\left(m_{2}\right)\right\} \\
& =\sum_{m_{2} n_{2} \in E_{2}, m_{1}=n_{1}} F_{N_{2}}\left(m_{2} n_{2}\right)+\sum_{m_{1} n_{1} \in E_{1}, m_{2}=n_{2}} F_{N_{1}}\left(m_{1} n_{1}\right) \\
& =(d)_{G_{2}}\left(m_{2}\right) F_{M_{1}}\left(m_{1}\right)+(d)_{G_{1}}\left(m_{1}\right) F_{M_{2}}\left(m_{2}\right),
\end{aligned}
$$

as claimed.

Example 3.11. Consider the vague graphs $\mathbf{G}_{1}, \mathbf{G}_{2}$, and $\mathbf{G}_{1} * \mathbf{G}_{2}$ as in Figure 9. Since $T_{M_{1}} \geq T_{N_{2}}, F_{M_{1}} \leq F_{N_{2}}, T_{M_{2}} \geq T_{N_{1}}$, and $F_{M_{2}} \leq F_{N_{1}}$, by Theorem 3.10 we have

$\left(d_{T}\right)_{\mathbf{G}_{1} * \mathbf{G}_{2}}(a, c)=(d)_{G_{2}}(c) T_{M_{1}}(a)+(d)_{G_{1}}(a) T_{M_{2}}(c)=1 \cdot(0.3)$ $+1 \cdot(0.2)=0.5$,

$\left(d_{F}\right)_{\mathbf{G}_{1} * \mathbf{G}_{2}}(a, c)=(d)_{G_{2}}(c) F_{M_{1}}(a)+(d)_{G_{1}}(a) F_{M_{2}}(c)=1 \cdot(0.4)$ $+1 \cdot(0.3)=0.7$.

$\left(d_{T}\right)_{\mathbf{G}_{1} * \mathbf{G}_{2}}(a, d)=(d)_{G_{2}}(d) T_{M_{1}}(a)+(d)_{G_{1}}(a) T_{M_{2}}(d)=1$.

$+1 \cdot(0.3)=0.6$,

$\left(d_{F}\right)_{\mathbf{G}_{1} * \mathbf{G}_{2}}(a, d)=(d)_{G_{2}}(d) F_{M_{1}}(a)+(d)_{G_{1}}(a) F_{M_{2}}(d)=1 \cdot$ $+1 \cdot(0.4)=0.8$.

$\left(d_{T}\right)_{\mathbf{G}_{1} * \mathbf{G}_{2}}(b, c)=(d)_{G_{2}}(c) T_{M_{1}}(b)+(d)_{G_{1}}(b) T_{M_{2}}(c)=1 \cdot(0.2)$ $+1 \cdot(0.2)=0.4$,

$\left(d_{F}\right)_{\mathbf{G}_{1} * \mathbf{G}_{2}}(b, c)=(d)_{G_{2}}(c) F_{M_{1}}(b)+(d)_{G_{1}}(b) F_{M_{2}}(c)=1 \cdot(0.3)$ $+1 \cdot(0.3)=0.6$. 
$\left(d_{T}\right)_{\mathbf{G}_{1} * \mathbf{G}_{2}}(b, d)=(d)_{G_{2}}(d) T_{M_{1}}(b)+(d)_{G_{1}}(b) T_{M_{2}}(d)=1 \cdot(0.2)$ $+1 \cdot(0.3)=0.5$,

$\left(d_{F}\right)_{\mathbf{G}_{1} * \mathbf{G}_{2}}(b, d)=(d)_{G_{2}}(d) F_{M_{1}}(b)+(d)_{G_{1}}(b) F_{M_{2}}(d)=1 \cdot(0.3)$ $+1 \cdot(0.4)=0.7$.

By direct calculations we obtain

$$
\begin{aligned}
\left(d_{T}\right)_{\mathbf{G}_{1} * \mathbf{G}_{2}}(a, c) & =0.3+0.2=0.5, \\
\left(d_{F}\right)_{\mathbf{G}_{1} * \mathbf{G}_{2}}(a, c) & =0.4+0.3=0.7, \\
\left(d_{T}\right)_{\mathbf{G}_{1} * \mathbf{G}_{2}}(a, d) & =0.3+0.3=0.6, \\
\left(d_{F}\right)_{\mathbf{G}_{1} * \mathbf{G}_{2}}(a, d) & =0.4+0.4=0.8, \\
\left(d_{T}\right)_{\mathbf{G}_{1} * \mathbf{G}_{2}}(b, c) & =0.2+0.2=0.4, \\
\left(d_{F}\right)_{\mathbf{G}_{1} * \mathbf{G}_{2}}(b, c) & =0.3+0.3=0.6, \\
\left(d_{T}\right)_{\mathbf{G}_{1} * \mathbf{G}_{2}}(b, d) & =0.3+0.2=0.5, \\
\left(d_{F}\right)_{\mathbf{G}_{1} * \mathbf{G}_{2}}(b, d) & =0.3+0.4=0.7 .
\end{aligned}
$$

It is clear that the degrees of vertices calculated using the formula in Theorem 3.10 and by the direct method are the same.

Definition 3.12. Let $\mathbf{G}_{1}=\left(M_{1}, N_{1}\right)$ and $\mathbf{G}_{2}=\left(M_{2}, N_{2}\right)$ be two vague graphs. For any vertex $\left(m_{1}, m_{2}\right) \in V_{1} \times V_{2}$ we define

$$
\begin{aligned}
\left(t d_{T}\right)_{\mathbf{G}_{1} * \mathbf{G}_{2}}\left(m_{1}, m_{2}\right)= & \sum_{\left(m_{1}, m_{2}\right)}\left(T_{\left.N_{1}, n_{2}\right) \in E_{1} \times E_{2}} * T_{N_{2}}\right)\left(\left(m_{1}, m_{2}\right)\left(n_{1}, n_{2}\right)\right) \\
+ & \left(T_{M_{1}} * T_{M_{2}}\right)\left(m_{1}, m_{2}\right) \\
= & \sum_{m_{1}=n_{1}, m_{2} n_{2} \in E_{2}} \max \left\{T_{M_{1}}\left(m_{1}\right), T_{N_{2}}\left(m_{2} n_{2}\right)\right\} \\
+ & \sum_{m_{1} n_{1} \in E_{1}, m_{2}=n_{2}} \max \left\{T_{N_{1}}\left(m_{1} n_{1}\right), T_{M_{2}}\left(m_{2}\right)\right\} \\
& \left.+\max _{M_{1}}\left(m_{1}\right), T_{M_{2}}\left(m_{2}\right)\right\}, \\
\left(t d_{F}\right)_{\mathbf{G}_{1} * \mathbf{G}_{2}}\left(m_{1}, m_{2}\right)= & \sum_{\left(m_{1}, m_{2}\right)\left(n_{1}, n_{2}\right) \in E_{1} \times E_{2}}\left(F_{N_{1}} * F_{N_{2}}\right)\left(\left(m_{1}, m_{2}\right)\left(n_{1}, n_{2}\right)\right) \\
+ & \left(F_{M_{1}} * F_{M_{2}}\right)\left(m_{1}, m_{2}\right) \\
= & \sum_{m_{1}=n_{1}, m_{2} n_{2} \in E_{2}} \min \left\{F_{M_{1}}\left(m_{1}\right), F_{N_{2}}\left(m_{2} n_{2}\right)\right\} \\
+ & \sum_{m_{1} \in E_{1}, m_{2}=n_{2}} \min \left\{F_{N_{1}}\left(m_{1} n_{1}\right), F_{M_{2}}\left(m_{2}\right)\right\} \\
& \left.+\min _{n_{1}}\left(m_{1}\right), F_{M_{2}}\left(m_{2}\right)\right\} .
\end{aligned}
$$

Example 3.13. In this example we find the degree and the total degree of vertices $(a, c)$ and $(a, d)$ in Example 3.2:

$$
\begin{aligned}
\left(d_{T}\right)_{\mathbf{G}_{1} * \mathbf{G}_{2}}(a, c) & =(d)_{G_{2}}(a) T_{M_{1}}(c)+(d)_{G_{1}}(c) T_{M_{2}}(a) \\
& =1(0.2)+4(0.4)=0.2+1.6=1.8 \\
\left(d_{F}\right)_{\mathbf{G}_{1} * \mathbf{G}_{2}}(a, c) & =(d)_{G_{2}}(a) F_{M_{1}}(c)+(d)_{G_{1}}(c) F_{M_{2}}(a) \\
& =1(0.5)+4(0.5)=0.3+1.2=1.5 .
\end{aligned}
$$

Therefore, $d_{\mathbf{G}_{1} * \mathbf{G}_{2}}(a, c)=(1.8,1.5)$. In addition, by the definition of the total vertex degree in the maximal product,

$$
\begin{aligned}
\left(t d_{T}\right)_{\mathbf{G}_{1} * \mathbf{G}_{2}}(a, c) & =(d)_{G_{2}}(a) T_{M_{1}}(c)+(d)_{G_{1}}(c) T_{M_{2}}(a) \\
& +\max \left\{T_{M_{1}}(a), T_{M_{2}}(c)\right\} \\
& =1(0.2)+4(0.4)+\max (0.2,0.4)=2.2 \\
\left(t d_{F}\right)_{\mathbf{G}_{1} * \mathbf{G}_{2}}(a, c) & =(d)_{G_{2}}(a) F_{M_{1}}(c)+(d)_{G_{1}}(c) F_{M_{2}}(a) \\
& +\min \left\{F_{M_{1}}(a), F_{M_{2}}(c)\right\} \\
& =1(0.5)+4(0.5)+\min (0.3,0.4)=1.8 .
\end{aligned}
$$

Therefore, $t d_{\mathbf{G}_{1} * \mathbf{G}_{2}}(a, c)=(2.2,1.8)$.

We also have

$$
\begin{aligned}
\left(d_{T}\right)_{\mathbf{G}_{1} * \mathbf{G}_{2}}(a, d) & =(d)_{G_{2}}(a) T_{M_{1}}(d)+(d)_{G_{1}}(d) T_{M_{2}}(a) \\
& =1(0.1)+4(0.4)=0.1+1.6=1.7 \\
\left(d_{F}\right)_{\mathbf{G}_{1} * \mathbf{G}_{2}}(a, d) & =(d)_{G_{2}}(a) F_{M_{1}}(d)+(d)_{G_{1}}(d) F_{M_{2}}(a) \\
& =1(0.3)+4(0.5)=0.3+2=2.3
\end{aligned}
$$

$$
\begin{aligned}
\left(t d_{T}\right)_{\mathbf{G}_{1} * \mathbf{G}_{2}}(a, d) & =(d)_{G_{2}}(a) T_{M_{1}}(d)+(d)_{G_{1}}(d) T_{M_{2}}(a) \\
& +\max \left\{T_{M_{1}}(a), T_{M_{2}}(d)\right\} \\
& =1(0.1)+4(0.4)+\max (0.4,0.1)=2.1,
\end{aligned}
$$

$$
\begin{aligned}
\left(t d_{F}\right)_{\mathbf{G}_{1} * \mathbf{G}_{2}}(a, d) & =(d)_{G_{2}}(a) F_{M_{1}}(d)+(d)_{G_{1}}(d) F_{M_{2}}(a) \\
& +\min \left\{F_{M_{1}}(a), F_{M_{2}}(d)\right\} \\
& =1(0.3)+4(0.5)+\min (0.5,0.3)=2.6
\end{aligned}
$$

Hence, $d_{\mathbf{G}_{1} * \mathbf{G}_{2}}(a, d)=(1.7,2.3)$ and $t d_{\mathbf{G}_{1} * \mathbf{G}_{2}}(a, d)=(2.1,2.6)$.

Similarly, we can find the degree and the total degree of all vertices in $\mathbf{G}_{1} * \mathbf{G}_{2}$.

Theorem 3.14. Let $\mathbf{G}_{1}=\left(M_{1}, N_{1}\right)$ and $\mathbf{G}_{2}=\left(M_{2}, N_{2}\right)$ be two vague graphs. If $T_{M_{1}} \geq T_{N_{2}}, F_{M_{1}} \leq F_{N_{2}}, T_{M_{2}} \geq T_{N_{1}}$, and $F_{M_{2}} \leq F_{N_{1}}$, then $\left(t d_{T}\right)_{\mathbf{G}_{1} * \mathbf{G}_{2}}\left(m_{1}, m_{2}\right)=(d)_{G_{2}}\left(m_{2}\right) T_{M_{1}}\left(m_{1}\right)+$ $(d)_{G_{1}}\left(m_{1}\right) T_{M_{2}}\left(m_{2}\right)+\max \left\{T_{M_{1}}\left(m_{1}\right), T_{M_{2}}\left(m_{2}\right)\right\} \quad$ and $\left(t d_{F}\right)_{\mathbf{G}_{1} * \mathbf{G}_{2}}\left(m_{1}, m_{2}\right)=(d)_{G_{2}}\left(m_{2}\right) F_{M_{1}}\left(m_{1}\right)+(d)_{G_{1}}\left(m_{1}\right) F_{M_{2}}\left(m_{2}\right)+$ $\min \left\{F_{M_{1}}\left(m_{1}\right), F_{M_{2}}\left(m_{2}\right)\right\}$.

Proof: From Definition 3.12 we have

$$
\begin{aligned}
\left(t d_{T}\right)_{\mathbf{G}_{1} * \mathbf{G}_{2}}\left(m_{1}, m_{2}\right)= & \sum_{\left(m_{1}, m_{2}\right)\left(n_{1}, n_{2}\right) \in E_{1} \times E_{2}}\left(T_{N_{1}} * T_{N_{2}}\right)\left(\left(m_{1}, m_{2}\right)\left(n_{1}, n_{2}\right)\right) \\
+ & \left(T_{M_{1}} * T_{M_{2}}\right)\left(m_{1}, m_{2}\right) \\
= & \sum_{m_{1}=n_{1}, m_{2} n_{2} \in E_{2}} \max \left\{T_{M_{1}}\left(m_{1}\right), T_{N_{2}}\left(m_{2} n_{2}\right)\right\} \\
+ & \sum m_{1} n_{1} \in E_{1}, m_{2}=n_{2} \\
& \left.+\max _{M_{1}}\left(m_{1}\right), T_{M_{2}}\left(m_{2}\right)\right\} \\
= & \left.\left.\sum_{N_{1}} T_{N_{2}}\left(m_{2} n_{2}\right)+\sum_{M_{1}}\right), T_{M_{2}}\left(m_{2}\right)\right\} \\
& m_{2} n_{2} \in E_{2}, m_{1}=n_{1} \\
& +\max \left\{T_{M_{1}}\left(m_{1}\right), T_{M_{2}}\left(m_{2}\right)\right\} \\
= & (d)_{G_{2}}\left(m_{2}\right) T_{M_{1}}\left(m_{1}\right)+(d)_{G_{1}}\left(m_{1}\right) T_{M_{2}}\left(m_{2}\right) \\
+ & \max \left\{T_{M_{1}}\left(m_{1}\right), T_{M_{2}}\left(m_{2}\right)\right\}
\end{aligned}
$$


and

$$
\begin{aligned}
\left(t d_{F}\right)_{\mathbf{G}_{1} * \mathbf{G}_{2}}\left(m_{1}, m_{2}\right)= & \sum_{\left(m_{1}, m_{2}\right)\left(n_{1}, n_{2}\right) \in E_{1} \times E_{2}}\left(F_{N_{1}} * F_{N_{2}}\right)\left(\left(m_{1}, m_{2}\right)\left(n_{1}, n_{2}\right)\right) \\
+ & \left(F_{M_{1}} * F_{M_{2}}\right)\left(m_{1}, m_{2}\right) \\
= & \sum_{m_{1}=n_{1}, m_{2} n_{2} \in E_{2}} \min \left\{F_{M_{1}}\left(m_{1}\right), F_{N_{2}}\left(m_{2} n_{2}\right)\right\} \\
+ & \sum \min \left\{F_{N_{1}}\left(m_{1} n_{1}\right), F_{M_{2}}\left(m_{2}\right)\right\} \\
& m_{1} n_{1} \in E_{1}, m_{2}=n_{2} \\
+ & \min \left\{F_{M_{1}}\left(m_{1}\right), F_{M_{2}}\left(m_{2}\right)\right\} \\
= & \sum_{m_{2} n_{2} \in E_{2}, m_{1}=n_{1}} F_{N_{2}}\left(m_{2} n_{2}\right)+\sum_{m_{1} n_{1} \in E_{1}, m_{2}=n_{2}} F_{N_{1}}\left(m_{1} n_{1}\right) \\
& +\min \left\{F_{M_{1}}\left(m_{1}\right), F_{M_{2}}\left(m_{2}\right)\right\} \\
= & (d)_{G_{2}}\left(m_{2}\right) F_{M_{1}}\left(m_{1}\right)+(d)_{G_{1}}\left(m_{1}\right) F_{M_{2}}\left(m_{2}\right) \\
& +\min \left\{F_{M_{1}}\left(m_{1}\right), F_{M_{2}}\left(m_{2}\right)\right\},
\end{aligned}
$$

as asserted.

Example 3.15. Consider the vague graphs $\mathbf{G}_{1}, \mathbf{G}_{2}$, and $\mathbf{G}_{1} * \mathbf{G}_{2}$ in Figure 9. The total degree of the vertex in the maximal product is calculated by the following formula:

$$
\begin{aligned}
\left(t d_{T}\right)_{\mathbf{G}_{1} * \mathbf{G}_{2}}\left(m_{1}, m_{2}\right)= & (d)_{G_{2}}\left(m_{2}\right) T_{M_{1}}\left(m_{1}\right)+(d)_{G_{1}}\left(m_{1}\right) T_{M_{2}}\left(m_{2}\right) \\
& +\max \left\{T_{M_{1}}\left(m_{1}\right), T_{M_{2}}\left(m_{2}\right)\right\} \\
\left(t d_{F}\right)_{\mathbf{G}_{1} * \mathbf{G}_{2}}\left(m_{1}, m_{2}\right)= & (d)_{G_{2}}\left(m_{2}\right) F_{M_{1}}\left(m_{1}\right)+(d)_{G_{1}}\left(m_{1}\right) F_{M_{2}}\left(m_{2}\right) \\
& +\min \left\{F_{M_{1}}\left(m_{1}\right), F_{M_{2}}\left(m_{2}\right)\right\}
\end{aligned}
$$

Using the formula we find that

$$
\begin{aligned}
\left(t d_{T}\right)_{\mathbf{G}_{1} * \mathbf{G}_{2}}(a, c) & =(d)_{G_{2}}(c) T_{M_{1}}(a)+(d)_{G_{1}}(a) T_{M_{2}}(c) \\
& +\max \left\{T_{M_{1}}(a), T_{M_{2}}(c)\right\} \\
& =1 \cdot(0.3)+1 \cdot(0.2)+\max \{0.2,0.3\} \\
& =0.3+0.2+0.3=0.8, \\
\left(t d_{F}\right)_{\mathbf{G}_{1} * \mathbf{G}_{2}}(a, c) & =(d)_{G_{2}}(c) F_{M_{1}}(a)+(d)_{G_{1}}(a) F_{M_{2}}(c) \\
& +\min \left\{F_{M_{1}}(a), F_{M_{2}}(c)\right\} \\
& =1 \cdot(0.4)+1 \cdot(0.3)+\min \{0.3,0.4\} \\
& =0.4+0.3+0.3=1 . \\
\left(t d_{T}\right)_{\mathbf{G}_{1} * \mathbf{G}_{2}}(a, d) & =(d)_{G_{2}}(d) T_{M_{1}}(a)+(d)_{G_{1}}(a) T_{M_{2}}(d) \\
& +\max \left\{T_{M_{1}}(a), T_{M_{2}}(d)\right\} \\
& =1 \cdot(0.3)+1 \cdot(0.3)+\max \{0.3,0.3\} \\
& =0.3+0.3+0.3=0.9, \\
\left(t d_{F}\right)_{\mathbf{G}_{1} * \mathbf{G}_{2}}(a, d) & =(d)_{G_{2}}(d) F_{M_{1}}(a)+(d)_{G_{1}}(a) F_{M_{2}}(d) \\
& +\min \left\{F_{M_{1}}(a), F_{M_{2}}(d)\right\} \\
& =1 \cdot(0.4)+1 \cdot(0.4)+\min \{0.4,0.4\} \\
& =0.4+0.4+0.4=1.2 .
\end{aligned}
$$

$$
\begin{aligned}
\left(t d_{T}\right)_{\mathbf{G}_{1} * \mathbf{G}_{2}}(b, c) & =(d)_{G_{2}}(c) T_{M_{1}}(b)+(d)_{G_{1}}(b) T_{M_{2}}(c) \\
& +\max \left\{T_{M_{1}}(b), T_{M_{2}}(c)\right\} \\
& =1 \cdot(0.2)+1 \cdot(0.2)+\max \{0.2,0.2\} \\
& =0.2+0.2+0.2=0.6, \\
\left(t d_{F}\right)_{\mathbf{G}_{1} * \mathbf{G}_{2}}(b, c) & =(d)_{G_{2}}(c) F_{M_{1}}(b)+(d)_{G_{1}}(b) F_{M_{2}}(c) \\
& +\min \left\{F_{M_{1}}(b), F_{M_{2}}(c)\right\} \\
& =1 \cdot(0.3)+1 \cdot(0.3)+\min \{0.3,0.3\} \\
& =0.3+0.3+0.3=0.9 . \\
\left(t d_{T}\right)_{\mathbf{G}_{1} * \mathbf{G}_{2}}(b, d) & =(d)_{G_{2}}(d) T_{M_{1}}(b)+(d)_{G_{1}}(b) T_{M_{2}}(d) \\
& +\max \left\{T_{M_{1}}(b), T_{M_{2}}(d)\right\} \\
& =1 \cdot(0.2)+1 \cdot(0.3)+\max \{0.2,0.3\} \\
& =0.2+0.3+0.3=0.8, \\
\left(t d_{F}\right)_{\mathbf{G}_{1} * \mathbf{G}_{2}}(b, d) & =(d)_{G_{2}}(d) F_{M_{1}}(b)+(d)_{G_{1}}(b) F_{M_{2}}(d) \\
& +\min \left\{F_{M_{1}}(b), F_{M_{2}}(d)\right\} \\
& =1 \cdot(0.3)+1 \cdot(0.4)+\min \{0.3,0.4\} \\
& =0.3+0.4+0.3=1 .
\end{aligned}
$$

On the other hand, by direct calculations we obtain

$$
\begin{aligned}
\left(t d_{T}\right)_{\mathbf{G}_{1} * \mathbf{G}_{2}}(a, c) & =0.3+0.2+0.3=0.8, \\
\left(t d_{F}\right)_{\mathbf{G}_{1} * \mathbf{G}_{2}}(a, c) & =0.4+0.3+0.3=1, \\
\left(t d_{T}\right)_{\mathbf{G}_{1} * \mathbf{G}_{2}}(a, d) & =0.3+0.3+0.3=0.9, \\
\left(t d_{F}\right)_{\mathbf{G}_{1} * \mathbf{G}_{2}}(a, d) & =0.4+0.4+0.4=1.2, \\
\left(t d_{T}\right)_{\mathbf{G}_{1} * \mathbf{G}_{2}}(b, c) & =0.2+0.2+0.2=0.6, \\
\left(t d_{F}\right)_{\mathbf{G}_{1} * \mathbf{G}_{2}}(b, c) & =0.3+0.3+0.3=0.9, \\
\left(t d_{T}\right)_{\mathbf{G}_{1} * \mathbf{G}_{2}}(b, d) & =0.3+0.2+0.3=0.8, \\
\left(t d_{F}\right)_{\mathbf{G}_{1} * \mathbf{G}_{2}}(b, d) & =0.4+0.3+0.3=1 .
\end{aligned}
$$

It is thus clear that the total degrees of vertices calculated using the formula and by the direct method are the same.

Definition 3.16. The rejection $\mathbf{G}_{1} \mid \mathbf{G}_{2}=\left(M_{1}\left|M_{2}, N_{1}\right| N_{2}\right)$ of two vague graphs $\mathbf{G}_{1}=\left(M_{1}, N_{1}\right)$ and $\mathbf{G}_{2}=\left(M_{2}, N_{2}\right)$ is defined as follows:

$$
\begin{aligned}
\left(T_{M_{1}} \mid T_{M_{2}}\right)\left(\left(m_{1}, m_{2}\right)\right)= & \min \left\{T_{M_{1}}\left(m_{1}\right), T_{M_{2}}\left(m_{2}\right)\right\}, \\
\left(F_{M_{1}} \mid F_{M_{2}}\right)\left(\left(m_{1}, m_{2}\right)\right)= & \max \left\{F_{M_{1}}\left(m_{1}\right), F_{M_{2}}\left(m_{2}\right)\right\} \\
& \forall\left(m_{1}, m_{2}\right) \in\left(V_{1} \times V_{2}\right) ;
\end{aligned}
$$

(ii) $\left(T_{N_{1}} \mid T_{N_{2}}\right)\left(\left(m, m_{2}\right)\left(m, n_{2}\right)\right)=\min \left\{T_{M_{1}}(m), T_{M_{2}}\left(m_{2}\right), T_{M_{2}}\left(n_{2}\right)\right\}$, $\left(F_{N_{1}} \mid F_{N_{2}}\right)\left(\left(m, m_{2}\right)\left(m, n_{2}\right)\right)=\max \left\{F_{M_{1}}(m), F_{M_{2}}\left(m_{2}\right), F_{M_{2}}\left(n_{2}\right)\right\}$

$$
\forall m \in V_{2} \text { and } m_{2} n_{2} \notin E_{2} \text {; }
$$

(iii) $\left(T_{N_{1}} \mid T_{N_{2}}\right)\left(\left(m, m_{2}\right)\left(m, n_{2}\right)\right)=\min \left\{T_{M_{1}}(m), T_{M_{2}}\left(m_{2}\right), T_{M_{2}}\left(n_{2}\right)\right\}$ $\left(F_{N_{1}} \mid F_{N_{2}}\right)\left(\left(m, m_{2}\right)\left(m, n_{2}\right)\right)=\max \left\{F_{M_{1}}(m), F_{M_{2}}\left(m_{2}\right), F_{M_{2}}\left(n_{2}\right)\right\}$ $\forall m \in V_{2}$ and $m_{1} n_{1} \notin E_{1}$;

(iv) $\left(T_{N_{1}} \mid T_{N_{2}}\right)\left(\left(m_{1}, m_{2}\right)\left(n_{1}, n_{2}\right)\right)=\min \left\{T_{M_{1}}\left(m_{1}\right), T_{M_{1}}\left(n_{1}\right), T_{M_{2}}\left(m_{2}\right), T_{M_{2}}\left(n_{2}\right)\right\}$, $\left(F_{N_{1}} \mid F_{N_{2}}\right)\left(\left(m_{1}, m_{2}\right)\left(n_{1}, n_{2}\right)\right)=\max \left\{F_{M_{1}}\left(m_{1}\right), F_{M_{1}}\left(n_{1}\right), F_{M_{2}}\left(m_{2}\right), F_{N_{2}}\left(n_{2}\right)\right\}$ $\forall m_{1} n_{1} \notin E_{1}$ and $m_{2} n_{2} \notin E_{2}$.

Example 3.17. Consider the vague graphs $\mathbf{G}_{1}$ and $\mathbf{G}_{2}$ in Figures 10,11 . The rejection of $\mathbf{G}_{1}$ and $\mathbf{G}_{2}$, i.e., $\mathbf{G}_{1} \mid \mathbf{G}_{2}$, is shown in Figure 12. 

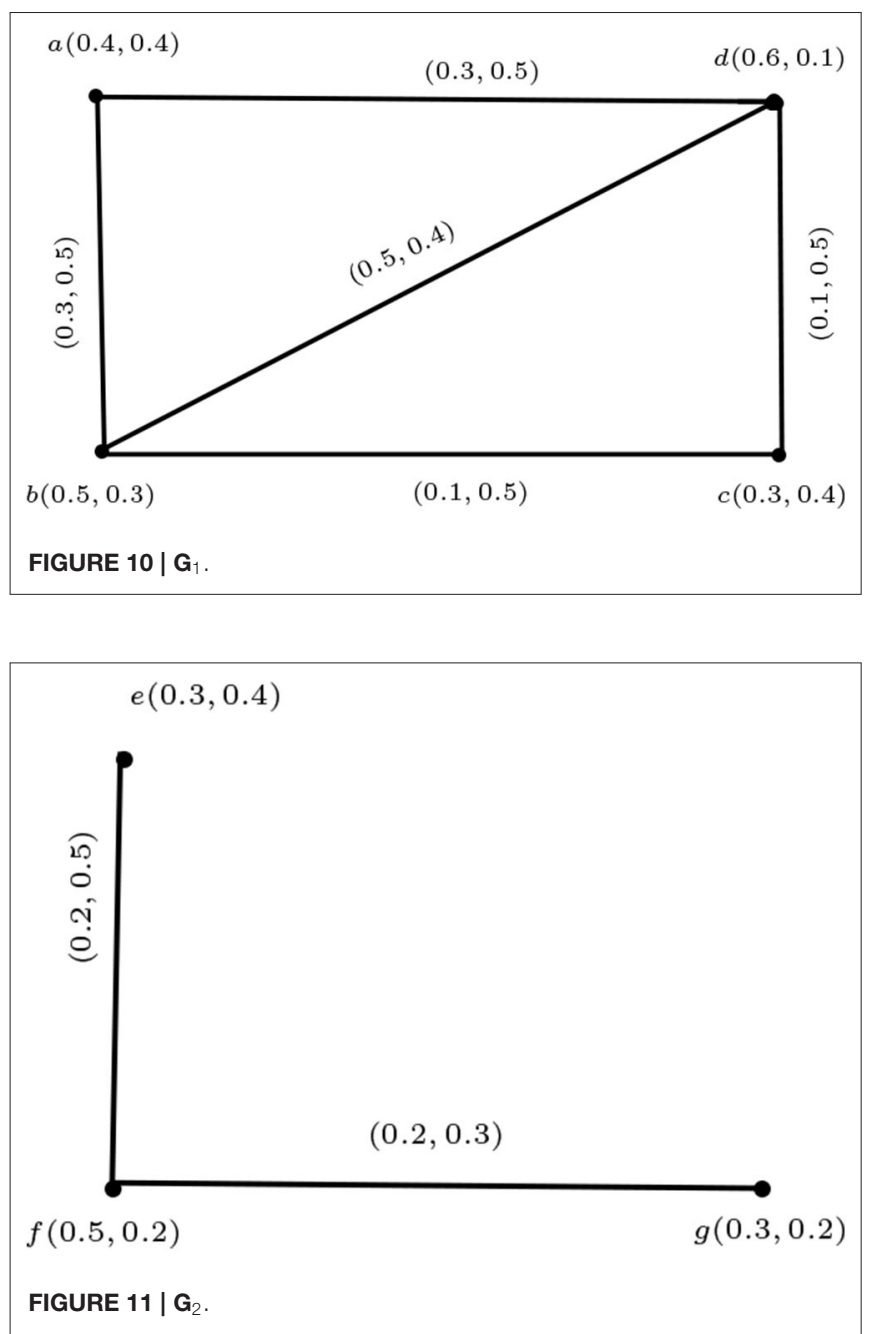

For the vertex $(a, e)$, we find the membership and nonmembership values as follows:

$$
\begin{aligned}
\left(T_{M_{1}} \mid T_{M_{2}}\right)((a, e)) & =\min \left\{T_{M_{1}}(a), T_{M_{2}}(e)\right\} \\
& =\min \{0.4,0.3\}=0.3 \\
\left(F_{M_{1}} \mid F_{M_{2}}\right)((a, e)) & =\max \left\{F_{M_{1}}(a), F_{M_{2}}(e)\right\} \\
& =\max \{0.4,0.4\}=0.4
\end{aligned}
$$

for $a \in V_{1}$ and $e \in V_{2}$.

For the edge $(e, c)(e, a)$, the membership and non-membership values are given by

$$
\begin{aligned}
\left(T_{N_{1}} \mid T_{N_{2}}\right)((e, c)(e, a)) & =\min \left\{T_{M_{1}}(e), T_{M_{2}}(c), T_{M_{2}}(a)\right\} \\
& =\min \{0.3,0.3,0.4\}=0.3, \\
\left(F_{N_{1}} \mid F_{N_{2}}\right)((e, c)(e, a)) & =\max \left\{F_{M_{1}}(e), F_{M_{2}}(c), F_{M_{2}}(a)\right\} \\
& =\max \{0.4,0.4,0.4\}=0.4
\end{aligned}
$$

for $e \in V_{2}$ and $a c \notin E_{1}$.
For the edge $(e, c)(e, g)$ we have

$$
\begin{aligned}
\left(T_{N_{1}} \mid T_{N_{2}}\right)((e, c)(e, g)) & =\min \left\{T_{M_{1}}(e), T_{M_{2}}(c), T_{M_{2}}(g)\right\} \\
& =\min \{0.3,0.3,0.3\}=0.3, \\
\left(F_{N_{1}} \mid F_{N_{2}}\right)((e, c)(e, g)) & =\max \left\{F_{M_{1}}(e), F_{M_{2}}(c), F_{M_{2}}(g)\right\} \\
& =\max \{0.4,0.4,0.2\}=0.4
\end{aligned}
$$

for $e \in V_{2}$ and $c g \notin E_{2}$.

Similarly, we can find the membership and non-membership values for all the remaining vertices and edges.

Proposition 3.18. The rejection of two vague graphs $\mathbf{G}_{1}$ and $\mathbf{G}_{2}$ is a vague graph.

Proof: Let $\mathbf{G}_{1}=\left(M_{1}, N_{1}\right)$ and $\mathbf{G}_{2}=\left(M_{2}, N_{2}\right)$ be two vague graphs on crisp graphs $G_{1}=\left(V_{1}, E_{1}\right)$ and $G_{2}=\left(V_{2}, E_{2}\right)$, respectively, and let $\left(\left(m_{1}, m_{2}\right)\left(n_{1}, n_{2}\right)\right) \in E_{1} \times E_{2}$. Then by Definition 3.16 we have the following:

(i) If $m_{1}=n_{1}$ and $m_{2} n_{2} \notin E_{2}$, then

$$
\begin{aligned}
& \left(T_{N_{1}} \mid T_{N_{2}}\right)\left(\left(m_{1}, m_{2}\right)\left(n_{1}, n_{2}\right)\right)=\min \left\{T_{M_{1}}\left(m_{1}\right), T_{M_{2}}\left(m_{2}\right), T_{M_{2}}\left(n_{2}\right)\right\} \\
& =\min \left\{\min \left\{T_{M_{1}}\left(m_{1}\right), T_{M_{2}}\left(m_{2}\right)\right\}, \min \left\{T_{M_{1}}\left(n_{1}\right), T_{M_{2}}\left(n_{2}\right)\right\}\right\} \\
& =\min \left\{\left(T_{M_{1}} \mid T_{M_{2}}\right)\left(m_{1}, m_{2}\right),\left(T_{M_{1}} \mid T_{M_{2}}\right)\left(n_{1}, n_{2}\right)\right\}, \\
& \left(F_{N_{1}} \mid F_{N_{2}}\right)\left(\left(m_{1}, m_{2}\right)\left(n_{1}, n_{2}\right)\right)=\max \left\{F_{M_{1}}\left(m_{1}\right), F_{M_{2}}\left(m_{2}\right), F_{M_{2}}\left(n_{2}\right)\right\} \\
& =\max \left\{\max \left\{F_{M_{1}}\left(m_{1}\right), F_{M_{2}}\left(m_{2}\right)\right\}, \max \left\{F_{M_{1}}\left(n_{1}\right), F_{M_{2}}\left(n_{2}\right)\right\}\right\} \\
& =\max \left\{\left(F_{M_{1}} \mid F_{M_{2}}\right)\left(m_{1}, m_{2}\right),\left(F_{M_{1}} \mid F_{M_{2}}\right)\left(n_{1}, n_{2}\right)\right\} .
\end{aligned}
$$

(ii) If $m_{2}=n_{2}$ and $m_{1} n_{1} \notin E_{1}$, then

$$
\begin{aligned}
& \left(T_{N_{1}} \mid T_{N_{2}}\right)\left(\left(m_{1}, m_{2}\right)\left(n_{1}, n_{2}\right)\right)=\min \left\{T_{M_{1}}\left(m_{1}\right), T_{M_{1}}\left(n_{1}\right), T_{M_{2}}\left(m_{2}\right)\right\} \\
& =\min \left\{\min \left\{T_{M_{1}}\left(m_{1}\right), T_{M_{2}}\left(m_{2}\right)\right\}, \min \left\{T_{M_{1}}\left(n_{1}\right), T_{M_{2}}\left(n_{2}\right)\right\}\right\} \\
& =\min \left\{\left(T_{M_{1}} \mid T_{M_{2}}\right)\left(m_{1}, m_{2}\right),\left(T_{M_{1}} \mid T_{M_{2}}\right)\left(n_{1}, n_{2}\right)\right\}, \\
& \left(F_{N_{1}} \mid F_{N_{2}}\right)\left(\left(m_{1}, m_{2}\right)\left(n_{1}, n_{2}\right)\right)=\max \left\{F_{M_{1}}\left(m_{1}\right), F_{M_{1}}\left(n_{1}\right), F_{M_{2}}\left(m_{2}\right)\right\} \\
& =\max \left\{\max \left\{F_{M_{1}}\left(m_{1}\right), F_{M_{2}}\left(m_{2}\right)\right\}, \max \left\{F_{M_{1}}\left(n_{1}\right), F_{M_{2}}\left(n_{2}\right)\right\}\right\} \\
& =\max \left\{\left(F_{M_{1}} \mid F_{M_{2}}\right)\left(m_{1}, m_{2}\right),\left(F_{M_{1}} \mid F_{M_{2}}\right)\left(n_{1}, n_{2}\right)\right\} .
\end{aligned}
$$

(iii) If $m_{1} n_{1} \notin E_{1}$ and $m_{2} n_{2} \notin E_{2}$, then

$$
\begin{aligned}
& \left(T_{N_{1}} \mid T_{N_{2}}\right)\left(\left(m_{1}, m_{2}\right)\left(n_{1}, n_{2}\right)\right)=\min \left\{T_{M_{1}}\left(m_{1}\right), T_{M_{1}}\left(n_{1}\right), T_{M_{2}}\left(m_{2}\right), T_{M_{2}}\left(n_{2}\right)\right\} \\
& =\min \left\{\min \left\{T_{M_{1}}\left(m_{1}\right), T_{M_{2}}\left(m_{2}\right)\right\}, \min \left\{T_{M_{1}}\left(n_{1}\right), T_{M_{2}}\left(n_{2}\right)\right\}\right\} \\
& =\min \left\{\left(T_{M_{1}} \mid T_{M_{2}}\right)\left(m_{1}, m_{2}\right),\left(T_{M_{1}} \mid T_{M_{2}}\right)\left(n_{1}, n_{2}\right)\right\}, \\
& \left(F_{N_{1}} \mid F_{N_{2}}\right)\left(\left(m_{1}, m_{2}\right)\left(n_{1}, n_{2}\right)\right)=\max \left\{F_{M_{1}}\left(m_{1}\right), F_{M_{1}}\left(n_{1}\right), F_{M_{2}}\left(m_{2}\right), T_{M_{2}}\left(n_{2}\right)\right\} \\
& =\max \left\{\max \left\{F_{M_{1}}\left(m_{1}\right), F_{M_{2}}\left(m_{2}\right)\right\}, \max \left\{F_{M_{1}}\left(n_{1}\right), F_{M_{2}}\left(n_{2}\right)\right\}\right\} \\
& =\max \left\{\left(F_{M_{1}} \mid F_{M_{2}}\right)\left(m_{1}, m_{2}\right),\left(F_{M_{1}} \mid F_{M_{2}}\right)\left(n_{1}, n_{2}\right)\right\} .
\end{aligned}
$$

Therefore, $\mathbf{G}_{1} \mid \mathbf{G}_{2}=\left(M_{1}\left|M_{2}, N_{1}\right| N_{2}\right)$ is a vague graph.

Remark 3.4. The rejection of two complete vague graphs $\mathbf{G}_{1}=\left(M_{1}, N_{1}\right)$ and $\mathbf{G}_{2}=\left(M_{2}, N_{2}\right)$ is a complete vague graph. 


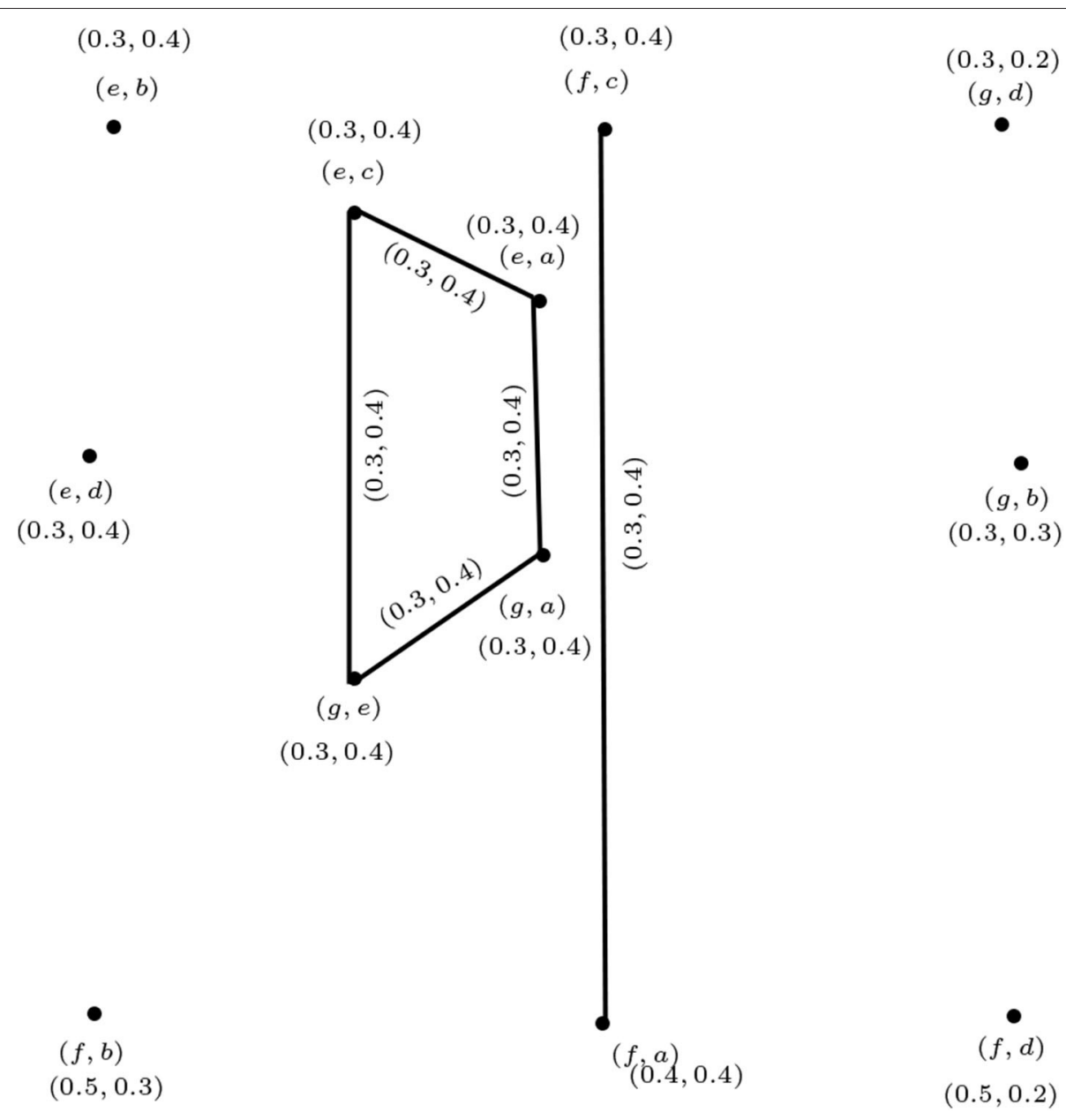

FIGURE $12\left|\mathbf{G}_{1}\right| \mathbf{G}_{2}$

Definition 3.19. Let $\mathbf{G}_{1}=\left(M_{1}, N_{1}\right)$ and $\mathbf{G}_{2}=\left(M_{2}, N_{2}\right)$ be two vague graphs. For any vertex $\left(m_{1}, m_{2}\right) \in V_{1} \times V_{2}$ we define

$$
\begin{aligned}
& \left(d_{T}\right)_{\mathbf{G}_{1} \mid \mathbf{G}_{2}}\left(m_{1}, m_{2}\right)=\sum_{\left(m_{1}, m_{2}\right)} \sum_{\left(n_{1}, n_{2}\right) \in E_{1} \times E_{2}}\left(T_{N_{1}} \mid T_{N_{2}}\right)\left(\left(m_{1}, m_{2}\right)\left(n_{1}, n_{2}\right)\right) \\
& =\sum_{m_{1}=n_{1}, m_{2} n_{2} \notin E_{2}} \min \left\{T_{M_{1}}\left(m_{1}\right), T_{M_{2}}\left(m_{2}\right), T_{M_{2}}\left(n_{2}\right)\right\} \\
& +\sum_{m_{2}=n_{2}, m_{1} n_{1} \notin E_{1}} \min \left\{T_{M_{1}}\left(m_{1}\right), T_{M_{1}}\left(n_{1}\right), T_{M_{2}}\left(m_{2}\right)\right\} \\
& +\sum_{m_{1} n_{1} \notin E_{1} \text { and } m_{2} n_{2} \notin E_{2}} \min \left\{T_{M_{1}}\left(m_{1}\right), T_{M_{1}}\left(n_{1}\right), T_{M_{2}}\left(m_{2}\right), T_{M_{2}}\left(n_{2}\right)\right\},
\end{aligned}
$$

$$
\begin{aligned}
& \left(d_{F}\right)_{\mathbf{G}_{1} \mid \mathbf{G}_{2}}\left(m_{1}, m_{2}\right)=\sum_{\left(m_{1}, m_{2}\right)\left(n_{1}, n_{2}\right) \in E_{1} \times E_{2}}\left(F_{N_{1}} \mid F_{N_{2}}\right)\left(\left(m_{1}, m_{2}\right)\left(n_{1}, n_{2}\right)\right) \\
& =\sum_{m_{1}=n_{1}, m_{2} n_{2} \notin E_{2}} \max \left\{F_{M_{1}}\left(m_{1}\right), F_{M_{2}}\left(m_{2}\right), F_{M_{2}}\left(n_{2}\right)\right\} \\
& +\sum_{m_{2}=n_{2}, m_{1} n_{1} \notin E_{1}} \max \left\{F_{M_{1}}\left(m_{1}\right), F_{M_{1}}\left(n_{1}\right), F_{M_{2}}\left(m_{2}\right)\right\} \\
& +\sum_{m_{1} n_{1} \notin E_{1} \text { and } m_{2} n_{2} \notin E_{2}} \max \left\{F_{M_{1}}\left(m_{1}\right), F_{M_{1}}\left(n_{1}\right), F_{M_{2}}\left(m_{2}\right), F_{M_{2}}\left(n_{2}\right)\right\} .
\end{aligned}
$$

Definition 3.20. Let $\mathbf{G}_{1}=\left(M_{1}, N_{1}\right)$ and $\mathbf{G}_{2}=\left(M_{2}, N_{2}\right)$ be two vague graphs. For any vertex $\left(m_{1}, m_{2}\right) \in V_{1} \times V_{2}$ we define

$$
\begin{aligned}
& \left(t d_{T}\right)_{\mathbf{G}_{1} \mid \mathbf{G}_{2}}\left(m_{1}, m_{2}\right)=\sum_{\left(m_{1}, m_{2}\right)\left(n_{1}, n_{2}\right) \in E_{1} \times E_{2}}\left(T_{N_{1}} \mid T_{N_{2}}\right)\left(\left(m_{1}, m_{2}\right)\left(n_{1}, n_{2}\right)\right) \\
& +\left(T_{M_{1}} \mid T_{M_{2}}\right)\left(m_{1}, m_{2}\right) \\
& =\sum_{m_{1}=n_{1}, m_{2} n_{2} \notin E_{2}} \min \left\{T_{M_{1}}\left(m_{1}\right), T_{M_{2}}\left(m_{2}\right), T_{M_{2}}\left(n_{2}\right)\right\} \\
& +\sum_{m_{2}=n_{2}, m_{1} n_{1} \notin E_{1}} \min \left\{T_{M_{1}}\left(m_{1}\right), T_{M_{1}}\left(n_{1}\right), T_{M_{2}}\left(m_{2}\right)\right\} \\
& +\sum_{m_{1} n_{1} \notin E_{1} \text { and } m_{2} n_{2} \notin E_{2}} \min \left\{T_{M_{1}}\left(m_{1}\right), T_{M_{1}}\left(n_{1}\right), T_{M_{2}}\left(m_{2}\right), T_{M_{2}}\left(n_{2}\right)\right\} \\
& +\left(d_{F}\right)_{\mathbf{G}_{1} \mid \mathbf{G}_{2}}\left(m_{1}, m_{2}\right)=\sum_{\left(m_{1}, m_{2}\right)\left(n_{1}, n_{2}\right) \in E_{1} \times E_{2}}\left(F_{N_{1}} \mid F_{N_{2}}\right)\left(\left(m_{1}, m_{2}\right)\left(n_{1}, n_{2}\right)\right) \\
& +\left(F_{M_{1}} \mid F_{M_{2}}\right)\left(m_{1}, m_{2}\right) \\
& =\sum_{m_{1}=n_{1}, m_{2} n_{2} \notin E_{2}} \max \left\{F_{M_{1}}\left(m_{1}\right), F_{M_{2}}\left(m_{2}\right), F_{M_{2}}\left(n_{2}\right)\right\} \\
& \quad+\sum_{m_{2}=n_{2}, m_{1} n_{1} \notin E_{1}} \max \left\{F_{M_{1}}\left(m_{1}\right), F_{M_{1}}\left(n_{1}\right), F_{M_{2}}\left(m_{2}\right)\right\} \\
& +\sum_{m_{1} n_{1} \notin E_{1} \text { and } m_{2} n_{2} \notin E_{2}} \max \left\{F_{M_{1}}\left(m_{1}\right), F_{M_{1}}\left(n_{1}\right), F_{M_{2}}\left(m_{2}\right), F_{M_{2}}\left(n_{2}\right)\right\} .
\end{aligned}
$$


Example 3.21. In this example we find the degree and total degree of the vertex $(e, a)$ in Example 3.17:

$$
\begin{aligned}
\left(d_{T}\right)_{\mathbf{G}_{1} \mid \mathbf{G}_{2}}(e, a) & =\min \left\{T_{M_{2}}(e), T_{M_{1}}(a), T_{M_{1}}(c)\right\} \\
& +\min \left\{T_{M_{2}}(e), T_{M_{1}}(a), T_{M_{2}}(g)\right\} \\
& =\min \{0.3,0.4,0.3\}+\min \{0.3,0.4,0.3\} \\
& =0.3+0.3 \\
& =0.6,
\end{aligned}
$$

$$
\begin{aligned}
\left(d_{F}\right)_{\mathbf{G}_{1} \mid \mathbf{G}_{2}}(e, a) & =\max \left\{F_{M_{2}}(e), F_{M_{1}}(a), F_{M_{1}}(c)\right\} \\
& +\max \left\{F_{M_{2}}(e), F_{M_{1}}(a), F_{M_{2}}(g)\right. \\
& =\max \{0.4,0.4,0.4\}+\max \{0.4,0.4,0.2\} \\
& =0.4+0.4 \\
& =0.8
\end{aligned}
$$

Therefore, $d_{\mathbf{G}_{1} \mid \mathbf{G}_{2}}(a, c)=(0.6,0.8)$.

In addition, by the definition of the total vertex degree in the maximal product,

$$
\begin{aligned}
\left(t d_{T}\right)_{\mathbf{G}_{1} \mid \mathbf{G}_{2}}(e, a)= & \min \left\{T_{M_{2}}(e), T_{M_{1}}(a), T_{M_{1}}(c)\right\} \\
+ & \min \left\{T_{M_{2}}(e), T_{M_{1}}(a), T_{M_{2}}(g)\right\} \\
& +\min \left\{T_{M_{2}}(e), T_{M_{1}}(a)\right\} \\
= & \min \{0.3,0.4,0.3\}+\min \{0.3,0.4,0.3\} \\
+ & \min \{0.3,0.4\} \\
= & 0.3+0.3+0.3 \\
= & 0.9
\end{aligned}
$$

$$
\begin{aligned}
\left(t d_{F}\right)_{\mathbf{G}_{1} \mid \mathbf{G}_{2}}(e, a)= & \max \left\{F_{M_{2}}(e), F_{M_{1}}(a), F_{M_{1}}(c)\right\} \\
+ & \max \left\{F_{M_{2}}(e), F_{M_{1}}(a), F_{M_{2}}(g)\right\} \\
& +\max \left\{F_{M_{2}}(e), F_{M_{1}}(a)\right\} \\
= & \max \{0.4,0.4,0.4\}+\max \{0.4,0.4,0.2\} \\
+ & \max \{0.3,0.4\} \\
= & 0.4+0.4+0.4 \\
= & 1.2 .
\end{aligned}
$$

Therefore, $t d_{\mathbf{G}_{1} \mid \mathbf{G}_{2}}(a, c)=(0.9,1.2)$.

Similarly, we can find the degree and the total degree of all vertices in $\mathbf{G}_{1} \mid \mathbf{G}_{2}$.

Definition 3.22. The symmetric difference $\mathbf{G}_{1} \oplus \mathbf{G}_{2}=\left(M_{1} \oplus\right.$ $\left.M_{2}, N_{1} \oplus N_{2}\right)$ of two vague graphs $\mathbf{G}_{1}=\left(M_{1}, N_{1}\right)$ and $\mathbf{G}_{2}=$
$\left(M_{2}, N_{2}\right)$ is defined as follows:

(i)

$$
\begin{aligned}
\left(T_{M_{1}} \oplus T_{M_{2}}\right)\left(\left(m_{1}, m_{2}\right)\right)= & \min \left\{T_{M_{1}}\left(m_{1}\right), T_{M_{2}}\left(m_{2}\right)\right\}, \\
\left(F_{M_{1}} \oplus F_{M_{2}}\right)\left(\left(m_{1}, m_{2}\right)\right)= & \max \left\{F_{M_{1}}\left(m_{1}\right), F_{M_{2}}\left(m_{2}\right)\right\} \\
& \forall\left(m_{1}, m_{2}\right) \in\left(V_{1} \times V_{2}\right) ;
\end{aligned}
$$

(ii) $\quad\left(T_{N_{1}} \oplus T_{N_{2}}\right)\left(\left(m, m_{2}\right)\left(m, n_{2}\right)\right)=\min \left\{T_{M_{1}}(m), T_{N_{2}}\left(m_{2} n_{2}\right)\right\}$,

$$
\left(F_{N_{1}} \oplus F_{N_{2}}\right)\left(\left(m, m_{2}\right)\left(m, n_{2}\right)\right)=\max \left\{F_{M_{1}}(m), F_{N_{2}}\left(m_{2} n_{2}\right)\right\}
$$

$\forall m \in V_{1}$ and $m_{2} n_{2} \in E_{2} ;$

(iii) $\quad\left(T_{N_{1}} \oplus T_{N_{2}}\right)\left(\left(m_{1}, z\right)\left(n_{1}, z\right)\right)=\min \left\{T_{N_{1}}\left(m_{1} n_{1}\right), T_{M_{2}}(z)\right\}$,

$\left(F_{N_{1}} \oplus F_{N_{2}}\right)\left(\left(m_{1}, z\right)\left(n_{1}, z\right)\right)=\max \left\{F_{N_{1}}\left(m_{1} n_{1}\right), F_{M_{2}}(z)\right\}$

$$
\forall z \in V_{2} \text { and } m_{1} n_{1} \in E_{1} \text {; }
$$

$$
\begin{aligned}
\text { (iv) }\left(T_{N_{1}} \oplus T_{N_{2}}\right)\left(\left(m_{1}, m_{2}\right)\left(n_{1}, n_{2}\right)\right)=\left\{\begin{array}{r}
\min \left\{T_{M_{1}}\left(m_{1}\right), T_{M_{1}}\left(n_{1}\right), T_{N_{2}}\left(m_{2} n_{2}\right)\right\} \\
\forall m_{1} n_{1} \notin E_{1} \text { and } m_{2} n_{2} \in E_{2}, \\
\min \left\{T_{M_{2}}\left(m_{2}\right), T_{M_{2}}\left(n_{2}\right), T_{N_{1}}\left(m_{1} n_{1}\right)\right\} \\
\forall m_{1} n_{1} \in E_{1} \text { and } m_{2} n_{2} \notin E_{2},
\end{array}\right. \\
\left(F_{N_{1}} \oplus F_{N_{2}}\right)\left(\left(m_{1}, m_{2}\right)\left(n_{1}, n_{2}\right)\right)=\left\{\begin{array}{r}
\max \left\{F_{M_{1}}\left(m_{1}\right), F_{M_{1}}\left(n_{1}\right), F_{N_{2}}\left(m_{2} n_{2}\right)\right\} \\
\forall m_{1} n_{1} \notin E_{1} \text { and } m_{2} n_{2} \in E_{2}, \\
\max \left\{F_{M_{2}}\left(m_{2}\right), F_{M_{2}}\left(n_{2}\right), F_{N_{1}}\left(m_{1} n_{1}\right)\right\} \\
\forall m_{1} n_{1} \in E_{1} \text { and } m_{2} n_{2} \notin E_{2} .
\end{array}\right.
\end{aligned}
$$

Example 3.23. Consider the vague graphs $\mathbf{G}_{1}$ and $\mathbf{G}_{2}$ as in Figures 13, 14. The symmetric difference of $\mathbf{G}_{1}$ and $\mathbf{G}_{2}$, i.e., $\mathbf{G}_{1} \oplus \mathbf{G}_{2}$, is shown in Figure $\mathbf{1 5}$.

For the vertex $(a, f)$, we find the membership and nonmembership values as follows:

$$
\begin{aligned}
\left(T_{M_{1}} \oplus T_{M_{2}}\right)((a, f)) & =\min \left\{T_{M_{1}}(a), T_{M_{2}}(f)\right\} \\
& =\min \{0.2,0.3\}=0.2, \\
\left(F_{M_{1}} \oplus F_{M_{2}}\right)((a, f)) & =\max \left\{F_{M_{1}}(a), F_{M_{2}}(f)\right\} \\
& =\max \{0.4,0.3\}=0.4
\end{aligned}
$$

for $a \in V_{1}$ and $f \in V_{2}$.

For the edge $(a, d)(a, e)$, the membership and non-membership values are given by

$$
\begin{aligned}
\left(T_{N_{1}} \oplus T_{N_{2}}\right)((a, d)(a, e)) & =\min \left\{T_{M_{1}}(a), T_{N_{2}}(d e)\right\} \\
& =\min \{0.2,0.1\}=0.1, \\
\left(F_{N_{1}} \oplus F_{N_{2}}\right)((a, d)(a, e)) & =\max \left\{F_{M_{1}}(a), F_{N_{2}}(d e)\right\} \\
& =\max \{0.4,0.6\}=0.6
\end{aligned}
$$

for $a \in V_{1}$ and $d e \in E_{2}$.

For the edge $(a, d)(b, d)$ we have

$$
\begin{aligned}
\left(T_{N_{1}} \oplus T_{N_{2}}\right)((a, d)(b, d)) & =\min \left\{T_{N_{1}}(a b), T_{M_{2}}(d)\right\} \\
& =\min \{0.2,0.2\}=0.2, \\
\left(F_{N_{1}} \oplus F_{N_{2}}\right)((a, d)(b, d)) & =\max \left\{F_{N_{1}}(a b), F_{M_{2}}(d)\right\} \\
& =\max \{0.7,0.4\}=0.7
\end{aligned}
$$

for $a b \in E_{1}$ and $d \in V_{2}$. 


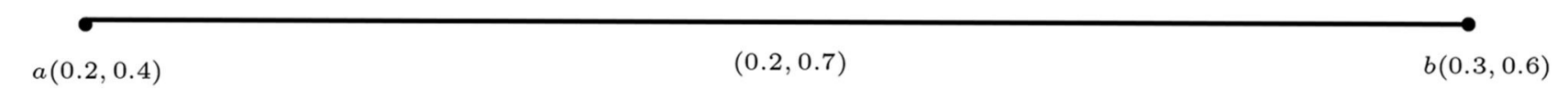

FIGURE 13 | $G_{1}$.
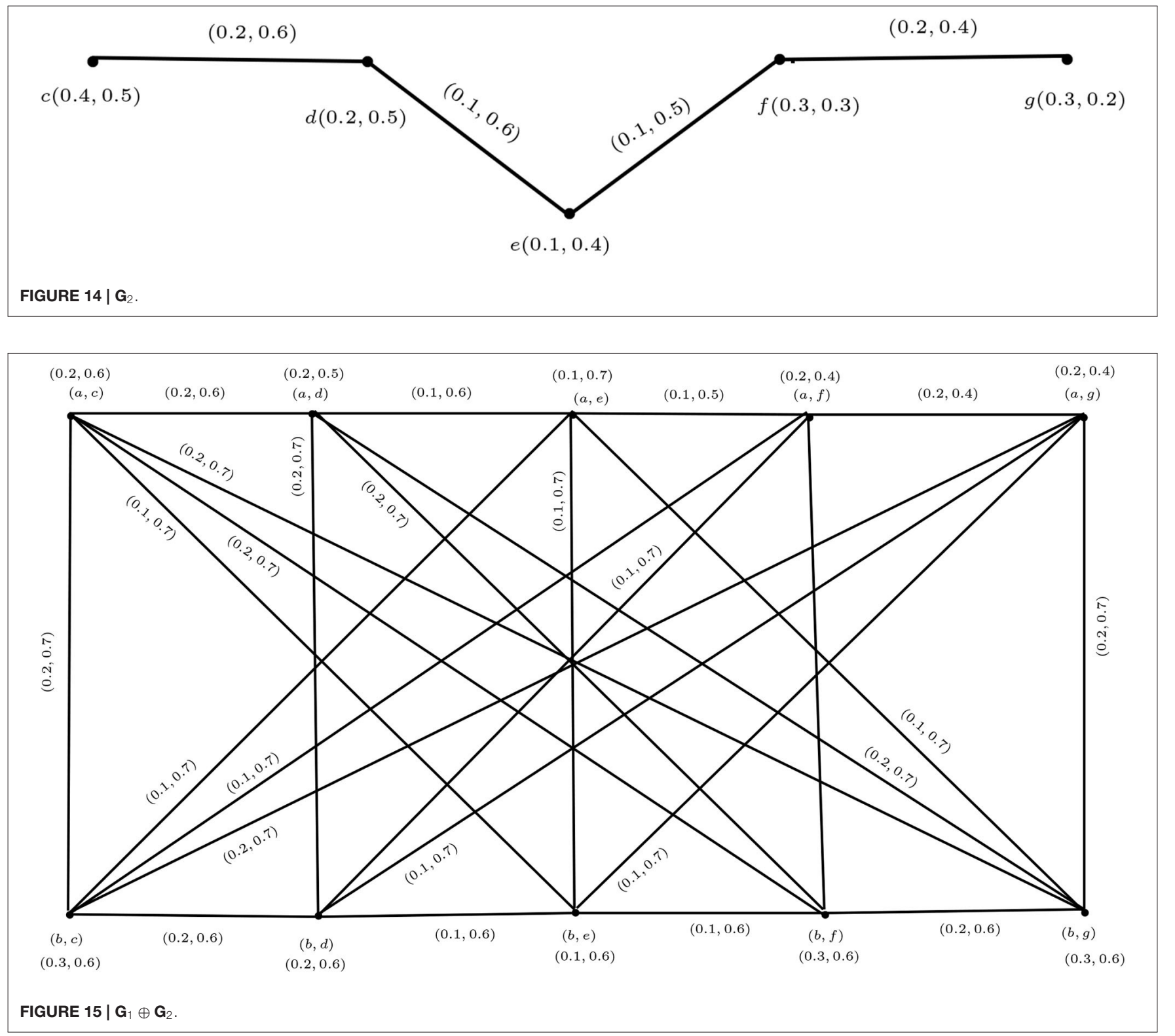

For the edge $(a, c)(b, f)$, the membership and non-membership values are

$$
\begin{aligned}
\left(T_{N_{1}} \oplus T_{N_{2}}\right)((a, c)(b, f)) & =\min \left\{T_{M_{2}}(c), T_{M_{2}}(f), T_{N_{1}}(a b)\right\} \\
& =\min \{0.4,0.3,0.2\}=0.2 \\
\left(F_{N_{1}} \oplus F_{N_{2}}\right)((a, c)(b, f)) & =\max \left\{F_{M_{2}}(c), F_{M_{2}}(f), F_{N_{1}}(a b)\right\} \\
& =\max \{0.5,0.3,0.7\}=0.7
\end{aligned}
$$

for $a b \in E_{1}$ and $c f \notin E_{2}$.
In the same way, we can find the membership and nonmembership values for all remaining vertices and edges.

Proposition 3.24. The symmetric difference of two vague graphs $\mathbf{G}_{1}$ and $\mathbf{G}_{2}$ is a vague graph.

Proof: Let $\mathbf{G}_{1}=\left(M_{1}, N_{1}\right)$ and $\mathbf{G}_{2}=\left(M_{2}, N_{2}\right)$ be two vague graphs on crisp graphs $G_{1}=\left(V_{1}, E_{1}\right)$ and $G_{2}=\left(V_{2}, E_{2}\right)$, respectively, and let $\left(\left(m_{1}, m_{2}\right)\left(n_{1}, n_{2}\right)\right) \in E_{1} \times E_{2}$. Then by Definition 3.22 we have the following cases: 
(i) If $m_{1}=n_{1}=m$, then

$\left(T_{N_{1}} \oplus T_{N_{2}}\right)\left(\left(m, m_{2}\right)\left(m, n_{2}\right)\right)=\min \left\{T_{M_{1}}(m), T_{N_{2}}\left(m_{2} n_{2}\right)\right\}$

$\leq \min \left\{T_{M_{1}}(m), \min \left\{T_{M_{2}}\left(m_{2}\right), T_{M_{2}}\left(n_{2}\right)\right\}\right\}$

$=\min \left\{\min \left\{T_{M_{1}}(m), T_{M_{2}}\left(m_{2}\right)\right\}, \min \left\{T_{M_{1}}(m), T_{M_{2}}\left(n_{2}\right)\right\}\right\}$

$=\min \left\{\left(T_{M_{1}} \oplus T_{M_{2}}\right)\left(m, m_{2}\right),\left(T_{M_{1}} \oplus T_{M_{2}}\right)\left(m, n_{2}\right)\right\}$,

$\left(F_{N_{1}} \oplus F_{N_{2}}\right)\left(\left(m, m_{2}\right)\left(m, n_{2}\right)\right)=\max \left\{F_{M_{1}}(m), F_{N_{2}}\left(m_{2} n_{2}\right)\right\}$

$\geq \max \left\{F_{M_{1}}(m), \max \left\{F_{M_{2}}\left(m_{2}\right), F_{M_{2}}\left(n_{2}\right)\right\}\right\}$

$=\max \left\{\max \left\{F_{M_{1}}(m), F_{M_{2}}\left(m_{2}\right)\right\}, \max \left\{F_{M_{1}}(m), F_{M_{2}}\left(n_{2}\right)\right\}\right\}$

$=\max \left\{\left(F_{M_{1}} \oplus F_{M_{2}}\right)\left(m, m_{2}\right),\left(F_{M_{1}} \oplus F_{M_{2}}\right)\left(m, n_{2}\right)\right\}$.

(ii) If $m_{2}=n_{2}=z$, then

$\left(T_{N_{1}} \oplus T_{N_{2}}\right)\left(\left(m_{1}, z\right)\left(n_{1}, z\right)\right)=\min \left\{T_{N_{1}}\left(m_{1} n_{1}\right), T_{M_{2}}(z)\right\}$

$\leq \min \left\{\min \left\{T_{N_{1}}\left(m_{1} n_{1}\right), T_{M_{2}}(z)\right\}\right.$

$=\min \left\{\min \left\{T_{M_{1}}\left(m_{1}\right), T_{M_{2}}(z)\right\}, \min \left\{T_{M_{1}}\left(n_{1}\right), T_{M_{2}}(z)\right\}\right\}$

$=\min \left\{\left(T_{M_{1}} \oplus T_{M_{2}}\right)\left(m_{1}, z\right),\left(T_{M_{1}} \oplus T_{M_{2}}\right)\left(n_{1}, z\right)\right\}$,

$\left(F_{N_{1}} \oplus F_{N_{2}}\right)\left(\left(m_{1}, z\right)\left(n_{1}, z\right)\right)=\max \left\{F_{N_{1}}\left(m_{1} n_{1}\right), F_{M_{2}}(z)\right\}$

$\geq \max \left\{\max \left\{F_{N_{1}}\left(m_{1} n_{1}\right), F_{M_{2}}(z)\right\}\right.$

$=\max \left\{\max \left\{F_{M_{1}}\left(m_{1}\right), F_{M_{2}}(z)\right\}, \max \left\{F_{M_{1}}\left(n_{1}\right), F_{M_{2}}(z)\right\}\right\}$

$=\max \left\{\left(F_{M_{1}} \oplus F_{M_{2}}\right)\left(m_{1}, z\right),\left(F_{M_{1}} \oplus F_{M_{2}}\right)\left(n_{1}, z\right)\right\}$.

(iii) If $m_{1} n_{1} \notin E_{1}$ and $m_{2} n_{2} \in E_{2}$, then

$\left(T_{N_{1}} \oplus T_{N_{2}}\right)\left(\left(m_{1}, m_{2}\right)\left(n_{1}, n_{2}\right)\right)=\min \left\{T_{M_{1}}\left(m_{1}\right), T_{M_{1}}\left(n_{1}\right), T_{N_{2}}\left(m_{2} n_{2}\right)\right\}$

$\leq \min \left\{T_{M_{1}}\left(m_{1}\right), T_{M_{1}}\left(n_{1}\right), \min \left\{T_{M_{2}}\left(m_{2}\right) T_{M_{2}}\left(n_{2}\right)\right\}\right\}$

$=\min \left\{\min \left\{T_{M_{1}}\left(m_{1}\right), T_{M_{2}}\left(m_{2}\right)\right\},\left\{T_{M_{1}}\left(m_{1}\right), T_{M_{2}}\left(n_{2}\right)\right\}\right\}$

$=\min \left\{\left(T_{M_{1}} \oplus T_{M_{2}}\right)\left(m_{1}, m_{2}\right),\left(T_{M_{1}} \oplus T_{M_{2}}\right)\left(n_{1}, n_{2}\right)\right\}$,

$\left(F_{N_{1}} \oplus F_{N_{2}}\right)\left(\left(m_{1}, m_{2}\right)\left(n_{1}, n_{2}\right)\right)=\max \left\{F_{M_{1}}\left(m_{1}\right), F_{M_{1}}\left(n_{1}\right), F_{N_{2}}\left(m_{2} n_{2}\right)\right\}$

$\geq \max \left\{F_{M_{1}}\left(m_{1}\right), F_{M_{1}}\left(n_{1}\right), \max \left\{F_{M_{2}}\left(m_{2}\right) F_{M_{2}}\left(n_{2}\right)\right\}\right\}$

$=\max \left\{\max \left\{F_{M_{1}}\left(m_{1}\right), F_{M_{2}}\left(m_{2}\right)\right\},\left\{F_{M_{1}}\left(m_{1}\right), F_{M_{2}}\left(n_{2}\right)\right\}\right\}$

$=\max \left\{\left(F_{M_{1}} \oplus F_{M_{2}}\right)\left(m_{1}, m_{2}\right),\left(F_{M_{1}} \oplus F_{M_{2}}\right)\left(n_{1}, n_{2}\right)\right\}$.

(iv) If $m_{1} n_{1} \in E_{1}$ and $m_{2} n_{2} \notin E_{2}$, then

$\left(T_{N_{1}} \oplus T_{N_{2}}\right)\left(\left(m_{1}, m_{2}\right)\left(n_{1}, n_{2}\right)\right)=\min \left\{T_{M_{2}}\left(m_{2}\right), T_{M_{2}}\left(n_{2}\right), T_{N_{1}}\left(m_{1} n_{1}\right)\right\}$

$\leq \min \left\{T_{M_{2}}\left(m_{2}\right), T_{M_{2}}\left(n_{2}\right), \min \left\{T_{M_{1}}\left(m_{1}\right) T_{M_{1}}\left(n_{1}\right)\right\}\right\}$

$=\min \left\{\min \left\{T_{M_{1}}\left(m_{1}\right), T_{M_{2}}\left(m_{2}\right)\right\},\left\{T_{M_{1}}\left(n_{1}\right), T_{M_{2}}\left(n_{2}\right)\right\}\right.$

$=\min \left\{\left(T_{M_{1}} \oplus T_{M_{2}}\right)\left(m_{1}, m_{2}\right),\left(T_{M_{1}} \oplus T_{M_{2}}\right)\left(n_{1}, n_{2}\right)\right\}$,

$\left(F_{N_{1}} \oplus F_{N_{2}}\right)\left(\left(m_{1}, m_{2}\right)\left(n_{1}, n_{2}\right)\right)=\max \left\{F_{M_{2}}\left(m_{2}\right), F_{M_{2}}\left(n_{2}\right), F_{N_{1}}\left(m_{1} n_{1}\right)\right\}$

$\geq \max \left\{F_{M_{2}}\left(m_{2}\right), F_{M_{2}}\left(n_{2}\right), \max \left\{F_{M_{1}}\left(m_{1}\right) F_{M_{1}}\left(n_{1}\right)\right\}\right\}$

$=\max \left\{\max \left\{F_{M_{2}}\left(m_{2}\right), F_{M_{1}}\left(m_{1}\right)\right\},\left\{F_{M_{2}}\left(m_{2}\right), F_{M_{1}}\left(n_{1}\right)\right\}\right.$

$=\max \left\{\left(F_{M_{1}} \oplus F_{M_{2}}\right)\left(m_{1}, m_{2}\right),\left(F_{M_{1}} \oplus F_{M_{2}}\right)\left(n_{1}, n_{2}\right)\right\}$.

Hence $\mathbf{G}_{1} \oplus \mathbf{G}_{2}$ is a vague graph.
Remark 3.5. The symmetric difference of two connected vague graphs $\mathbf{G}_{1}=\left(M_{1}, N_{1}\right)$ and $\mathbf{G}_{2}=\left(M_{2}, N_{2}\right)$ is connected, because we include the case where $\left(m_{1}, m_{2}\right) \in E_{1}$ and $\left(n_{1}, n_{2}\right) \in E_{2}$ in the definition of the symmetric difference of two vague graphs.

Definition 3.25. Let $\mathbf{G}_{1}=\left(M_{1}, N_{1}\right)$ and $\mathbf{G}_{2}=\left(M_{2}, N_{2}\right)$ be two vague graphs. For any vertex $\left(m_{1}, m_{2}\right) \in V_{1} \times V_{2}$ we define

$$
\begin{aligned}
\left(d_{T}\right)_{\mathbf{G}_{1} \oplus \mathbf{G}_{2}}\left(m_{1}, m_{2}\right)= & \sum_{\left(m_{1}, m_{2}\right)\left(n_{1}, n_{2}\right) \in E_{1} \times E_{2}}\left(T_{N_{1}} \oplus T_{N_{2}}\right)\left(\left(m_{1}, m_{2}\right)\left(n_{1}, n_{2}\right)\right) \\
= & \sum_{m_{1}=n_{1}, m_{2} n_{2} \in E_{2}} \min \left\{T_{M_{1}}\left(m_{1}\right), T_{N_{2}}\left(m_{2} n_{2}\right)\right\} \\
& +\sum_{m_{1} n_{1} \in E_{1}, m_{2}=n_{2}} \min \left\{T_{N_{1}}\left(m_{1} n_{1}\right), T_{M_{2}}\left(m_{2}\right)\right\} \\
& +\sum_{m_{1} \notin E_{1} \text { and } m_{2} n_{2} \in E_{2}} \min \left\{T_{M_{1}}\left(m_{1}\right), T_{M_{1}}\left(n_{1}\right), T_{N_{2}}\left(m_{2} n_{2}\right)\right\} \\
& +\sum_{m_{1} n_{1} \in E_{1} \text { and } m_{2} n_{2} \notin E_{2}} \min \left\{T_{N_{1}}\left(m_{1} n_{1}\right), T_{M_{2}}\left(m_{2}\right), T_{M_{2}}\left(n_{2}\right)\right\}
\end{aligned}
$$

and

$$
\begin{aligned}
\left(d_{F}\right)_{\mathbf{G}_{1} \oplus \mathbf{G}_{2}}\left(m_{1}, m_{2}\right)= & \sum_{\left(m_{1}, m_{2}\right)\left(n_{1}, n_{2}\right) \in E_{1} \times E_{2}}\left(F_{N_{1}} \oplus F_{N_{2}}\right)\left(\left(m_{1}, m_{2}\right)\left(n_{1}, n_{2}\right)\right) \\
= & \sum_{m_{1}=n_{1}, m_{2} n_{2} \in E_{2}} \max \left\{F_{M_{1}}\left(m_{1}\right), F_{N_{2}}\left(m_{2} n_{2}\right)\right\} \\
& +\sum_{m_{1} n_{1} \in E_{1}, m_{2}=n_{2}} \max \left\{F_{N_{1}}\left(m_{1} n_{1}\right), F_{M_{2}}\left(m_{2}\right)\right\} \\
& +\sum_{m_{1} n_{1} \notin E_{1} \text { and } m_{2} n_{2} \in E_{2}} \max \left\{F_{M_{1}}\left(m_{1}\right), F_{M_{1}}\left(n_{1}\right), F_{N_{2}}\left(m_{2} n_{2}\right)\right\} \\
& +\sum_{m_{1} n_{1} \in E_{1} \text { and } m_{2} n_{2} \notin E_{2}} \max \left\{F_{N_{1}}\left(m_{1} n_{1}\right), F_{M_{2}}\left(m_{2}\right), F_{M_{2}}\left(n_{2}\right)\right\} .
\end{aligned}
$$

Theorem 3.26. Let $\mathbf{G}_{1}=\left(M_{1}, N_{1}\right)$ and $\mathbf{G}_{2}=\left(M_{2}, N_{2}\right)$ be two vague graphs. If $T_{M_{1}} \geq T_{N_{2}}, F_{M_{1}} \leq F_{N_{2}}, T_{M_{2}} \geq T_{N_{1}}$, and $F_{M_{2}} \leq F_{N_{1}}$, then for every $\left(m_{1}, m_{2}\right) \in V_{1} \times V_{2}$ we have $(d)_{\mathbf{G}_{1} \oplus \mathbf{G}_{2}}\left(m_{1}, m_{2}\right)=q(d)_{\mathbf{G}_{1}}\left(m_{1}\right)+s(d)_{\mathbf{G}_{2}}\left(m_{2}\right)$, where $s=\left|V_{1}\right|-$ $(d)_{G_{1}}\left(m_{1}\right)$ and $q=\left|V_{2}\right|-(d)_{G_{2}}\left(m_{2}\right)$.

Proof: Using Definition 3.25,

$$
\begin{aligned}
\left(d_{T}\right)_{\mathbf{G}_{1} \oplus \mathbf{G}_{2}}\left(m_{1}, m_{2}\right)= & \sum_{\left(m_{1}, m_{2}\right)\left(n_{1}, n_{2}\right) \in E_{1} \times E_{2}}\left(T_{N_{1}} \oplus T_{N_{2}}\right)\left(\left(m_{1}, m_{2}\right)\left(n_{1}, n_{2}\right)\right) \\
= & \sum_{m_{1}=n_{1}, m_{2} n_{2} \in E_{2}} \min \left\{T_{M_{1}}\left(m_{1}\right), T_{N_{2}}\left(m_{2} n_{2}\right)\right\} \\
& +\sum_{m_{1} n_{1} \in E_{1}, m_{2}=n_{2}} \min \left\{T_{N_{1}}\left(m_{1} n_{1}\right), T_{M_{2}}\left(m_{2}\right)\right\} \\
& +\sum_{m_{1} n_{1} \notin E_{1} \text { and } m_{2} n_{2} \in E_{2}} \min \left\{T_{M_{1}}\left(m_{1}\right), T_{M_{1}}\left(n_{1}\right), T_{N_{2}}\left(m_{2} n_{2}\right)\right\} \\
& +\sum_{m_{1} n_{1} \in E_{1} \text { and } m_{2} n_{2} \notin E_{2}} \min \left\{T_{N_{1}}\left(m_{1} n_{1}\right), T_{M_{2}}\left(m_{2}\right), T_{M_{2}}\left(n_{2}\right)\right\} \\
& =\sum_{m_{2} n_{2} \in E_{2}} T_{N_{2}}\left(m_{2} n_{2}\right)+\sum_{m_{1} n_{1} \in E_{1}} T_{N_{1}}\left(m_{1} n_{1}\right) \\
& \left.+\sum_{m_{1} n_{1} \notin E_{1} \text { and } m_{2} n_{2} \in E_{2}} T_{N_{2}}\left(m_{2} n_{2}\right)\right\}+\sum_{m_{1} n_{1} \in E_{1} \text { and } m_{2} n_{2} \notin E_{2}} T_{N_{1}}\left(m_{1} n_{1}\right) \\
& =q\left(d_{T}\right)_{\mathbf{G}_{1}}\left(m_{1}\right)+s\left(d_{T}\right)_{\mathbf{G}_{2}}\left(m_{2}\right),
\end{aligned}
$$




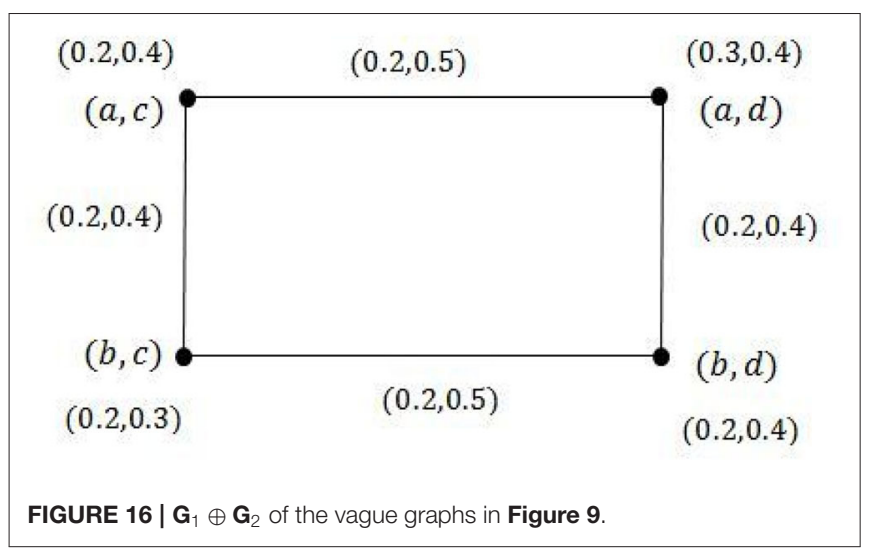

$$
\begin{aligned}
\left(d_{F}\right)_{\mathbf{G}_{1} \oplus \mathbf{G}_{2}}\left(m_{1}, m_{2}\right)= & \sum_{\left(m_{1}, m_{2}\right)\left(n_{1}, n_{2}\right) \in E_{1} \times E_{2}}\left(F_{N_{1}} \oplus F_{N_{2}}\right)\left(\left(m_{1}, m_{2}\right)\left(n_{1}, n_{2}\right)\right) \\
= & \sum_{m_{1}=n_{1}, m_{2} n_{2} \in E_{2}} \max \left\{F_{M_{1}}\left(m_{1}\right), F_{N_{2}}\left(m_{2} n_{2}\right)\right\} \\
& +\sum_{m_{1} n_{1} \in E_{1}, m_{2}=n_{2}} \max \left\{F_{N_{1}}\left(m_{1} n_{1}\right), F_{M_{2}}\left(m_{2}\right)\right\} \\
& +\sum_{m_{1} n_{1} \notin E_{1} \text { and } m_{2} n_{2} \in E_{2}} \max \left\{F_{M_{1}}\left(m_{1}\right), F_{M_{1}}\left(n_{1}\right), F_{N_{2}}\left(m_{2} n_{2}\right)\right\} \\
& +\sum_{n_{1} \in E_{1} \text { and } m_{2} n_{2} \notin E_{2}} \max \left\{F_{N_{1}}\left(m_{1} n_{1}\right), F_{M_{2}}\left(m_{2}\right), F_{M_{2}}\left(n_{2}\right)\right\} \\
& =\sum_{m_{2} n_{2} \in E_{2}} F_{N_{2}}\left(m_{2} n_{2}\right)+\sum_{m_{1} n_{1} \in E_{1}} F_{N_{1}}\left(m_{1} n_{1}\right) \\
& \left.+\sum_{m_{1} n_{1} \notin E_{1} \text { and } m_{2} n_{2} \in E_{2}} F_{N_{2}}\left(m_{2} n_{2}\right)\right\}+m_{1} \sum_{N_{1} \in E_{1} \text { and } m_{2} n_{2} \notin E_{2}}\left(m_{1} n_{1}\right) \\
& =q\left(d_{F}\right) \mathbf{G}_{1}\left(m_{1}\right)+s\left(d_{F}\right) \mathbf{G}_{2}\left(m_{2}\right),
\end{aligned}
$$

and hence the result is proved.

Example 3.27. Consider the two vague graphs $\mathbf{G}_{1}$ and $\mathbf{G}_{2}$ in Figure 9 and their symmetric difference in Figure 16. In Figure 9, $T_{M_{1}} \geq T_{N_{2}}, F_{M_{1}} \leq F_{N_{2}}, T_{M_{2}} \geq T_{N_{1}}$, and $F_{M_{2}} \leq F_{N_{1}}$. Then, the total degree of a vertex in the symmetric difference is calculated by the following formula:

$$
\begin{array}{r}
\left(d_{T}\right)_{\mathbf{G}_{1} \oplus \mathbf{G}_{2}}\left(m_{1}, m_{2}\right)=q\left(d_{T}\right)_{\mathbf{G}_{1}}\left(m_{1}\right)+s\left(d_{T}\right)_{\mathbf{G}_{2}}\left(m_{2}\right), \\
\left(d_{F}\right)_{\mathbf{G}_{1} \oplus \mathbf{G}_{2}}\left(m_{1}, m_{2}\right)=q\left(d_{F}\right)_{\mathbf{G}_{1}}\left(m_{1}\right)+s\left(d_{F}\right)_{\mathbf{G}_{2}}\left(m_{2}\right) .
\end{array}
$$

Using the formula we find that

$$
\begin{aligned}
\left(d_{T}\right)_{\mathbf{G}_{1} \oplus \mathbf{G}_{2}}(a, c) & =1 \cdot(0.2)+1 \cdot(0.2)=0.4, \\
\left(d_{F}\right)_{\mathbf{G}_{1} \oplus \mathbf{G}_{2}}(a, c) & =1 \cdot(0.4)+1 \cdot(0.5)=0.9, \\
\left(d_{T}\right)_{\mathbf{G}_{1} \oplus \mathbf{G}_{2}}(a, d) & =1 \cdot(0.2)+1 \cdot(0.2)=0.4, \\
\left(d_{F}\right)_{\mathbf{G}_{1} \oplus \mathbf{G}_{2}}(a, d) & =1 \cdot(0.4)+1 \cdot(0.5)=0.9 .
\end{aligned}
$$

Hence, $(d)_{\mathbf{G}_{1} \oplus \mathbf{G}_{2}}(a, c)=(0.4,0.9)$ and $(d)_{\mathbf{G}_{1} \oplus \mathbf{G}_{2}}(a, d)=$ $(0.4,0.9)$.
In the same way, we can show that $(d)_{\mathbf{G}_{1} \oplus \mathbf{G}_{2}}(b, c)=$ $(d)_{\mathbf{G}_{1} \oplus \mathbf{G}_{2}}(b, d)=(0.4,0.9)$. Direct calculations give

$$
\begin{aligned}
\left(d_{T}\right)_{\mathbf{G}_{1} \oplus \mathbf{G}_{2}}(a, c) & =0.2+0.2=0.4, \\
\left(d_{F}\right)_{\mathbf{G}_{1} \oplus \mathbf{G}_{2}}(a, c) & =0.4+0.5=0.9, \\
\left(d_{T}\right)_{\mathbf{G}_{1} \oplus \mathbf{G}_{2}}(a, d) & =0.2+0.2=0.4, \\
\left(d_{F}\right)_{\mathbf{G}_{1} \oplus \mathbf{G}_{2}}(a, d) & =0.4+0.5=0.9, \\
\left(d_{T}\right)_{\mathbf{G}_{1} \oplus \mathbf{G}_{2}}(b, c) & =0.2+0.2=0.4, \\
\left(d_{F}\right)_{\mathbf{G}_{1} \oplus \mathbf{G}_{2}}(b, c) & =0.4+0.5=0.9, \\
\left(d_{T}\right)_{\mathbf{G}_{1} \oplus \mathbf{G}_{2}}(b, d) & =0.2+0.2=0.4, \\
\left(d_{F}\right)_{\mathbf{G}_{1} \oplus \mathbf{G}_{2}}(b, d) & =0.4+0.5=0.9 .
\end{aligned}
$$

It is obvious from the above that the degrees of vertices calculated using the formula and by the direct method are the same.

Definition 3.28. Let $\mathbf{G}_{1}=\left(M_{1}, N_{1}\right)$ and $\mathbf{G}_{2}=\left(M_{2}, N_{2}\right)$ be two vague graphs. For any vertex $\left(m_{1}, m_{2}\right) \in V_{1} \times V_{2}$ we define

$$
\begin{aligned}
& \left(t d_{T}\right)_{\mathbf{G}_{1} \oplus \mathbf{G}_{2}}\left(m_{1}, m_{2}\right)=\sum_{\left(m_{1}, m_{2}\right)}\left(T_{\left.n_{1}, n_{2}\right) \in E_{1} \times E_{2}} \oplus T_{N_{2}}\right)\left(\left(m_{1}, m_{2}\right)\left(n_{1}, n_{2}\right)\right) \\
& +\left(T_{M_{1}} \oplus T_{M_{2}}\left(m_{1}, m_{2}\right)\right. \\
& =\sum_{m_{1}=n_{1}, m_{2} n_{2} \in E_{2}} \min \left\{T_{M_{1}}\left(m_{1}\right), T_{N_{2}}\left(m_{2} n_{2}\right)\right\} \\
& +\sum_{m_{1} n_{1} \in E_{1}, m_{2}=n_{2}} \min \left\{T_{N_{1}}\left(m_{1} n_{1}\right), T_{M_{2}}\left(m_{2}\right)\right\} \\
& +\sum_{m_{1} n_{1} \notin E_{1} \text { and } m_{2} n_{2} \in E_{2}} \min \left\{T_{M_{1}}\left(m_{1}\right), T_{M_{1}}\left(n_{1}\right), T_{N_{2}}\left(m_{2} n_{2}\right)\right\} \\
& +\sum_{m_{1} n_{1} \in E_{1} \text { and } m_{2} n_{2} \notin E_{2}} \min \left\{T_{N_{1}}\left(m_{1} n_{1}\right), T_{M_{2}}\left(m_{2}\right), T_{M_{2}}\left(n_{2}\right)\right\} \\
& +\min \left\{T_{M_{1}}\left(m_{1}\right), T_{M_{2}}\left(m_{2}\right)\right\}
\end{aligned}
$$

and

$$
\begin{aligned}
& \left(t d_{F}\right)_{\mathbf{G}_{1} \oplus \mathbf{G}_{2}}\left(m_{1}, m_{2}\right)=\sum_{\left(m_{1}, m_{2}\right)\left(n_{1}, n_{2}\right) \in E_{1} \times E_{2}}\left(F_{N_{1}} \oplus F_{N_{2}}\right)\left(\left(m_{1}, m_{2}\right)\left(n_{1}, n_{2}\right)\right) \\
& +\left(F_{M_{1}} \oplus F_{M_{2}}\left(m_{1}, m_{2}\right)\right. \\
& =\sum_{m_{1}=n_{1}, m_{2} n_{2} \in E_{2}} \max \left\{F_{M_{1}}\left(m_{1}\right), F_{N_{2}}\left(m_{2} n_{2}\right)\right\} \\
& +\sum_{m_{1} n_{1} \in E_{1}, m_{2}=n_{2}} \max \left\{F_{N_{1}}\left(m_{1} n_{1}\right), F_{M_{2}}\left(m_{2}\right)\right\} \\
& +\sum_{m_{1} n_{1} \notin E_{1} \text { and } m_{2} n_{2} \in E_{2}} \max \left\{F_{M_{1}}\left(m_{1}\right), F_{M_{1}}\left(n_{1}\right), F_{N_{2}}\left(m_{2} n_{2}\right)\right\} \\
& +\sum_{m_{1} n_{1} \in E_{1} \text { and } m_{2} n_{2} \notin E_{2}} \max \left\{F_{N_{1}}\left(m_{1} n_{1}\right), F_{M_{2}}\left(m_{2}\right), F_{M_{2}}\left(n_{2}\right)\right\} \\
& +\max \left\{F_{M_{1}}\left(m_{1}\right), F_{M_{2}}\left(m_{2}\right)\right\} .
\end{aligned}
$$

Example 3.29. In this example we find the degree and total degree of the vertex $(a, e)$ in Example 3.23. We have

$$
\begin{aligned}
s & =\left|V_{1}\right|-(d)_{G_{1}}(a) \\
& =2-1=1
\end{aligned}
$$


and, similarly,

$$
\begin{aligned}
q & =\left|V_{2}\right|-(d)_{G_{2}}(e) \\
& =5-2=3 .
\end{aligned}
$$

Therefore

$$
\begin{aligned}
\left(d_{T}\right)_{\mathbf{G}_{1} \oplus \mathbf{G}_{2}}(a, e) & =q\left(d_{T}\right)_{\mathbf{G}_{1}}(a)+s\left(d_{T}\right)_{\mathbf{G}_{2}}(e) \\
& =3(0.2)+1(0.1+0.1)=0.6+0.2=0.8, \\
\left(d_{F}\right)_{\mathbf{G}_{1} \oplus \mathbf{G}_{2}}(a, e) & =q\left(d_{F}\right)_{\mathbf{G}_{1}}(a)+s\left(d_{F}\right)_{\mathbf{G}_{2}}(e) \\
& =3(0.4)+1(0.6+0.5)=1.2+1.1=2.3 .
\end{aligned}
$$

So

$$
(d)_{\mathbf{G}_{1} \oplus \mathbf{G}_{2}}(a, e)=(0.8,2.3) .
$$

In addition, by Definition 3.28 we have

$$
\begin{aligned}
\left(t d_{T}\right)_{\mathbf{G}_{1} \oplus \mathbf{G}_{2}}(a, e) & =q\left(t d_{T}\right)_{\mathbf{G}_{1}}(a)+s\left(t d_{T}\right)_{\mathbf{G}_{2}}(e) \\
& -(s-1) T_{\mathbf{G}_{2}}(e)-(q-1) T_{\mathbf{G}_{1}}(a) \\
& -\max \left\{T_{\mathbf{G}_{1}}(a), T_{\mathbf{G}_{2}}(e)\right\} \\
& =3(0.2+0.2)+1(0.1+0.1+0.1) \\
& -(1-1)(0.1)-(3-1)(0.2)-\max \{0.2,0.1\} \\
& =3(0.4)+0.3-0.4-0.2=0.9, \\
\left(t d_{F}\right)_{\mathbf{G}_{1} \oplus \mathbf{G}_{2}}(a, e) & =q\left(t d_{F}\right)_{\mathbf{G}_{1}}(a)+s\left(t d_{F}\right)_{\mathbf{G}_{2}}(e) \\
& -(s-1) F_{\mathbf{G}_{2}}(e)-(q-1) F_{\mathbf{G}_{1}}(a) \\
& -\min \left\{F_{\mathbf{G}_{1}}(a), F_{\mathbf{G}_{2}}(e)\right\} \\
& =3(0.2+0.2)+1(0.4+0.5+0.6) \\
& -(1-1)(0.4)-(3-1)(0.4)-\min \{0.4,0.4\} \\
& =3(0.4)+1.5-0.8-0.4=1.5 .
\end{aligned}
$$

Therefore,

$$
(t d)_{\mathbf{G}_{1} \oplus \mathbf{G}_{2}}(a, e)=(0.9,1.5) .
$$

Similarly, we can find the degree and total degree of all vertices in $\mathbf{G}_{1} \oplus \mathbf{G}_{2}$.

Theorem 3.30. Let $\mathbf{G}_{1}=\left(M_{1}, N_{1}\right)$ and $\mathbf{G}_{2}=\left(M_{2}, N_{2}\right)$ be two vague graphs.

(i) If $T_{M_{1}} \geq T_{N_{2}}$ and $T_{M_{2}} \geq T_{N_{1}}$, then for all $\left(m_{1}, m_{2}\right) \in$ $V_{1} \times V_{2}$,

$$
\begin{gathered}
\left(t d_{T}\right)_{\mathbf{G}_{1} \oplus \mathbf{G}_{2}}\left(m_{1}, m_{2}\right)=q\left(t d_{T}\right)_{\mathbf{G}_{1}}\left(m_{1}\right)+s\left(t d_{T}\right)_{\mathbf{G}_{2}}\left(m_{2}\right) \\
-(q-1) T_{\mathbf{G}_{1}}\left(m_{1}\right)-\max \left\{T_{\mathbf{G}_{1}}\left(m_{1}\right), T_{\mathbf{G}_{2}}\left(m_{2}\right)\right\} .
\end{gathered}
$$

(ii) If $F_{M_{1}} \leq F_{N_{2}}$ and $F_{M_{2}} \leq F_{N_{1}}$, then for all $\left(m_{1}, m_{2}\right) \in$ $V_{1} \times V_{2}$,

$$
\begin{gathered}
\left(t d_{F}\right)_{\mathbf{G}_{1} \oplus \mathbf{G}_{2}}\left(m_{1}, m_{2}\right)=q\left(t d_{F}\right)_{\mathbf{G}_{1}}\left(m_{1}\right)+s\left(t d_{F}\right)_{\mathbf{G}_{2}}\left(m_{2}\right) \\
-(q-1) F_{\mathbf{G}_{1}}\left(m_{1}\right)-\min \left\{F_{\mathbf{G}_{1}}\left(m_{1}\right), F_{\mathbf{G}_{2}}\left(m_{2}\right)\right\} .
\end{gathered}
$$

Here $s=\left|V_{1}\right|-(d)_{G_{1}}\left(m_{1}\right)$ and $q=\left|V_{2}\right|-(d)_{G_{2}}\left(m_{2}\right)$.
Proof: For all $\left(m_{1}, m_{2}\right) \in V_{1} \times V_{2}$ we have

$$
\begin{aligned}
& \left(t d_{T}\right)_{\mathbf{G}_{1} \oplus \mathbf{G}_{2}}\left(m_{1}, m_{2}\right)=\sum_{\left(m_{1}, m_{2}\right)\left(n_{1}, n_{2}\right) \in E_{1} \times E_{2}}\left(T_{N_{1}} \oplus T_{N_{2}}\right)\left(\left(m_{1}, m_{2}\right)\left(n_{1}, n_{2}\right)\right) \\
& +\left(T_{M_{1}} \oplus T_{M_{2}}\right)\left(m_{1}, m_{2}\right) \\
& =\sum_{m_{1}=n_{1}, m_{2} n_{2} \in E_{2}} \min \left\{T_{M_{1}}\left(m_{1}\right), T_{N_{2}}\left(m_{2} n_{2}\right)\right\} \\
& +\sum_{m_{1} n_{1} \in E_{1}, m_{2}=n_{2}} \min \left\{T_{N_{1}}\left(m_{1} n_{1}\right), T_{M_{2}}\left(m_{2}\right)\right\} \\
& +\sum_{m_{1} n_{1} \notin E_{1} \text { and } m_{2} n_{2} \in E_{2}} \min \left\{T_{M_{1}}\left(m_{1}\right), T_{M_{1}}\left(n_{1}\right), T_{N_{2}}\left(m_{2} n_{2}\right)\right\} \\
& +\sum_{m_{1} n_{1} \in E_{1} \text { and } m_{2} n_{2} \notin E_{2}} \min \left\{T_{N_{1}}\left(m_{1} n_{1}\right), T_{M_{2}}\left(m_{2}\right), T_{M_{2}}\left(n_{2}\right)\right\} \\
& +\max \left\{T_{M_{1}}\left(m_{1}\right), T_{M_{2}}\left(m_{2}\right)\right\} \\
& =\sum_{m_{2} n_{2} \in E_{2}} T_{N_{2}}\left(m_{2} n_{2}\right)+\sum_{m_{1} n_{1} \in E_{1}} T_{N_{1}}\left(m_{1} n_{1}\right) \\
& \left.+\sum_{m_{1} n_{1} \notin E_{1} \text { and } m_{2} n_{2} \in E_{2}} T_{N_{2}}\left(m_{2} n_{2}\right)\right\}+\sum_{m_{1} n_{1} \in E_{1} \text { and } m_{2} n_{2} \notin E_{2}} T_{N_{1}}\left(m_{1} n_{1}\right) \\
& +\max \left\{T_{M_{1}}\left(m_{1}\right), T_{M_{2}}\left(m_{2}\right)\right\} \\
& =\sum_{m_{2} n_{2} \in E_{2}} T_{N_{2}}\left(m_{2} n_{2}\right)+\sum_{m_{1} n_{1} \in E_{1}} T_{N_{1}}\left(m_{1} n_{1}\right) \\
& \left.+\sum_{m_{1} n_{1} \notin E_{1} \text { and } m_{2} n_{2} \in E_{2}} T_{N_{2}}\left(m_{2} n_{2}\right)\right\} \\
& +\sum_{m_{1} n_{1} \in E_{1} \text { and } m_{2} n_{2} \notin E_{2}} T_{N_{1}}\left(m_{1} n_{1}\right)+T_{M_{1}}\left(m_{1}\right)+T_{M_{2}}\left(m_{2}\right) \\
& -\max \left\{T_{M_{1}}\left(m_{1}\right), T_{M_{2}}\left(m_{2}\right)\right\} \\
& =q\left(t d_{T}\right)_{\mathbf{G}_{1}}\left(m_{1}\right)+s\left(t d_{T}\right)_{\mathbf{G}_{2}}\left(m_{2}\right) \\
& -(q-1) T_{\mathbf{G}_{1}}\left(m_{1}\right)-\max \left\{T_{\mathbf{G}_{1}}\left(m_{1}\right), T_{\mathbf{G}_{2}}\left(m_{2}\right)\right\},
\end{aligned}
$$

$$
\begin{aligned}
& \left(t d_{F}\right)_{\mathbf{G}_{1} \oplus \mathbf{G}_{2}}\left(m_{1}, m_{2}\right)=\sum_{\substack{\left(m_{1}, m_{2}\right)\left(n_{1}, n_{2}\right) \in E_{1} \times E_{2} \\
+\left(F_{M_{1}} \oplus F_{M_{2}}\right)\left(m_{1}, m_{2}\right)}}\left(F_{N_{1}} \oplus F_{N_{2}}\right)\left(\left(m_{1}, m_{2}\right)\left(n_{1}, n_{2}\right)\right) \\
& =\sum_{m_{1}=n_{1}, m_{2} n_{2} \in E_{2}} \max \left\{F_{M_{1}}\left(m_{1}\right), F_{N_{2}}\left(m_{2} n_{2}\right)\right\} \\
& \quad+\sum_{m_{1} n_{1} \in E_{1}, m_{2}=n_{2}} \max \left\{F_{N_{1}}\left(m_{1} n_{1}\right), F_{M_{2}}\left(m_{2}\right)\right\} \\
& \quad+\sum_{m_{1} n_{1} \notin E_{1} \text { and } m_{2} n_{2} \in E_{2}} \max \left\{F_{M_{1}}\left(m_{1}\right), F_{M_{1}}\left(n_{1}\right), F_{N_{2}}\left(m_{2} n_{2}\right)\right\} \\
& \quad+\sum_{m_{1} n_{1} \in E_{1} \text { and } m_{2} n_{2} \notin E_{2}} \max \left\{F_{N_{1}}\left(m_{1} n_{1}\right), F_{M_{2}}\left(m_{2}\right), F_{M_{2}}\left(n_{2}\right)\right\} \\
& \quad+\sum_{m_{2} n_{2} \in E_{2}} F_{N_{2}}\left(m_{2} n_{2}\right)+\sum_{m_{1} n_{1} \in E_{1}} F_{N_{1}}\left(m_{1} n_{1}\right) \\
& \left.\quad+\sum_{m_{1} n_{1} \notin E_{1} \text { and } m_{2} n_{2} \in E_{2}} F_{N_{2}}\left(m_{2} n_{2}\right)\right\}
\end{aligned}
$$




$$
\begin{aligned}
& +\sum_{m_{1} n_{1} \in E_{1} \text { and } m_{2} n_{2} \notin E_{2}} F_{N_{1}}\left(m_{1} n_{1}\right) \\
& +\min \left\{F_{M_{1}}\left(m_{1}\right), F_{M_{2}}\left(m_{2}\right)\right\} \\
& =\sum_{m_{2} n_{2} \in E_{2}} F_{N_{2}}\left(m_{2} n_{2}\right)+\sum_{m_{1} n_{1} \in E_{1}} F_{N_{1}}\left(m_{1} n_{1}\right) \\
& \left.+\sum_{m_{1} n_{1} \notin E_{1} \text { and } m_{2} n_{2} \in E_{2}} F_{N_{2}}\left(m_{2} n_{2}\right)\right\} \\
& +\sum_{m_{1} n_{1} \in E_{1} \text { and } m_{2} n_{2} \notin E_{2}} F_{N_{1}}\left(m_{1} n_{1}\right)+F_{M_{1}}\left(m_{1}\right)+F_{M_{2}}\left(m_{2}\right) \\
& =q\left(t d_{F}\right)_{\mathbf{G}_{1}}\left(m_{1}\right)+s\left(t d_{F}\right)_{\mathbf{G}_{2}}\left(m_{2}\right) \\
& \quad-(q-1) F_{\mathbf{G}_{1}}\left(m_{1}\right)-\min \left\{F_{M_{1}}\left(m_{1}\right), F_{M_{2}}\left(m_{2}\right)\right\}
\end{aligned}
$$

where $s=\left|V_{1}\right|-(d)_{G_{1}}\left(m_{1}\right)$ and $q=\left|V_{2}\right|-(d)_{G_{2}}\left(m_{2}\right)$.

Example 3.31. In this example, we calculate the total degree of the vertices in Example 3.27.

The total degree of a vertex in the symmetric difference is given by

$$
\begin{aligned}
& \left(t d_{T}\right)_{\mathbf{G}_{1} \oplus \mathbf{G}_{2}}\left(m_{1}, m_{2}\right)=q\left(t d_{T}\right)_{\mathbf{G}_{1}}\left(m_{1}\right)+s\left(t d_{T}\right)_{\mathbf{G}_{2}}\left(m_{2}\right) \\
& -(q-1) T_{\mathbf{G}_{1}}\left(m_{1}\right)-\max \left\{T_{\mathbf{G}_{1}}\left(m_{1}\right), T_{\mathbf{G}_{2}}\left(m_{2}\right)\right\}, \\
& \left(t d_{F}\right)_{\mathbf{G}_{1} \oplus \mathbf{G}_{2}}\left(m_{1}, m_{2}\right)=q\left(t d_{F}\right)_{\mathbf{G}_{1}}\left(m_{1}\right)+s\left(t d_{F}\right)_{\mathbf{G}_{2}}\left(m_{2}\right) \\
& -(q-1) F_{\mathbf{G}_{1}}\left(m_{1}\right)-\min \left\{F_{\mathbf{G}_{1}}\left(m_{1}\right), F_{\mathbf{G}_{2}}\left(m_{2}\right)\right\} .
\end{aligned}
$$

Using the above formula, we calculate

$$
\begin{aligned}
\left(t d_{T}\right)_{\mathbf{G}_{1} \oplus \mathbf{G}_{2}}(a, c) & =1 \cdot(0.5)+1 \cdot(0.4)-(1-1) \cdot(0.3) \\
& -\max \{0.2,0.3\}=0.6 \\
\left(t d_{F}\right)_{\mathbf{G}_{1} \oplus \mathbf{G}_{2}}(a, c) & =1 \cdot(0.8)+1 \cdot(0.8)-(1-1) \cdot(0.4) \\
& -\min \{0.3,0.4\}=1.3, \\
\left(t d_{T}\right)_{\mathbf{G}_{1} \oplus \mathbf{G}_{2}}(a, d) & =1 \cdot(0.5)+1 \cdot(0.5)-(1-1) \cdot(0.3) \\
& -\max \{0.3,0.3\}=0.7 \\
\left(t d_{F}\right)_{\mathbf{G}_{1} \oplus \mathbf{G}_{2}}(a, d) & =1 \cdot(0.8)+1 \cdot(0.9)-(1-1) \cdot(0.4) \\
& -\min \{0.4,0.4\}=1.3, \\
\left(t d_{T}\right)_{\mathbf{G}_{1} \oplus \mathbf{G}_{2}}(b, c) & =1 \cdot(0.4)+1 \cdot(0.4)-(1-1) \cdot(0.2) \\
& -\max \{0.2,0.2\}=0.6, \\
\left(t d_{F}\right)_{\mathbf{G}_{1} \oplus \mathbf{G}_{2}}(b, c) & =1 \cdot(0.7)+1 \cdot(0.8)-(1-1) \cdot(0.2) \\
& -\min \{0.3,0.3\}=1.2, \\
\left(t d_{T}\right)_{\mathbf{G}_{1} \oplus \mathbf{G}_{2}}(b, d) & =1 \cdot(0.4)+1 \cdot(0.5)-(1-1) \cdot(0.2) \\
& -\max \{0.2,0.3\}=0.6, \\
\left(t d_{F}\right)_{\mathbf{G}_{1} \oplus \mathbf{G}_{2}}(b, d) & =1 \cdot(0.7)+1 \cdot(0.9)-(1-1) \cdot(0.2) \\
& -\min \{0.3,0.4\}=1.3 .
\end{aligned}
$$

By direct calculations, we find

$$
\begin{aligned}
\left(t d_{T}\right)_{\mathbf{G}_{1} \oplus \mathbf{G}_{2}}(a, c) & =0.2+0.2+0.2=0.6, \\
\left(t d_{F}\right)_{\mathbf{G}_{1} \oplus \mathbf{G}_{2}}(a, c) & =0.4+0.5+0.4=1.3, \\
\left(t d_{T}\right)_{\mathbf{G}_{1} \oplus \mathbf{G}_{2}}(a, d) & =0.2+0.2+0.3=0.7, \\
\left(t d_{F}\right)_{\mathbf{G}_{1} \oplus \mathbf{G}_{2}}(a, d) & =0.4+0.5+0.4=1.3, \\
\left(t d_{T}\right)_{\mathbf{G}_{1} \oplus \mathbf{G}_{2}}(b, c) & =0.2+0.2+0.2=0.6, \\
\left(t d_{F}\right)_{\mathbf{G}_{1} \oplus \mathbf{G}_{2}}(b, c) & =0.4+0.5+0.3=1.2, \\
\left(t d_{T}\right)_{\mathbf{G}_{1} \oplus \mathbf{G}_{2}}(b, d) & =0.2+0.2+0.2=0.6, \\
\left(t d_{F}\right)_{\mathbf{G}_{1} \oplus \mathbf{G}_{2}}(b, d) & =0.4+0.5+0.4=1.3 .
\end{aligned}
$$

It is clear that the total degrees of vertices calculated using the formula and by the direct method are the same.

Definition 3.32. The residue product $\mathbf{G}_{1} \bullet \mathbf{G}_{2}=\left(M_{1} \bullet M_{2}, N_{1} \bullet\right.$ $\left.N_{2}\right)$ of two vague graphs $\mathbf{G}_{1}=\left(M_{1}, N_{1}\right)$ and $\mathbf{G}_{2}=\left(M_{2}, N_{2}\right)$ is defined as follows:

$$
\begin{aligned}
\left(T_{M_{1}} \bullet T_{M_{2}}\right)\left(\left(m_{1}, m_{2}\right)\right) & =\max \left\{T_{M_{1}}\left(m_{1}\right), T_{M_{2}}\left(m_{2}\right)\right\}, \\
\left(F_{M_{1}} \bullet F_{M_{2}}\right)\left(\left(m_{1}, m_{2}\right)\right) & =\min \left\{F_{M_{1}}\left(m_{1}\right), F_{M_{2}}\left(m_{2}\right)\right\} \\
& \forall\left(m_{1}, m_{2}\right) \in\left(V_{1} \times V_{2}\right) ;
\end{aligned}
$$

(ii) $\quad\left(T_{N_{1}} \bullet T_{N_{2}}\right)\left(\left(m_{1}, m_{2}\right)\left(n_{1}, n_{2}\right)\right)=T_{N_{1}}\left(m_{1} n_{1}\right)$,

$$
\begin{aligned}
\left(F_{N_{1}} \bullet F_{N_{2}}\right)\left(\left(m_{1}, m_{2}\right)\left(n_{1}, n_{2}\right)\right) & =F_{N_{1}}\left(m_{1} n_{1}\right) \\
& \forall m_{1} n_{1} \in E_{1}, m_{2} \neq n_{2} .
\end{aligned}
$$

Example 3.33. Consider the vague graphs $\mathbf{G}_{1}$ and $\mathbf{G}_{2}$ in Figures 17,18 . The residue product of $\mathbf{G}_{1}$ and $\mathbf{G}_{2}$, i.e., $\mathbf{G}_{1} \bullet \mathbf{G}_{2}$, is shown in Figure 19.

For the vertex $(b, e)$, we find the membership and nonmembership values as follows:

$$
\begin{aligned}
\left(T_{M_{1}} \bullet T_{M_{2}}\right)((b, e)) & =\max \left\{T_{M_{1}}(b), T_{M_{2}}(e)\right\} \\
& =\max \{0.2,0.2\}=0.2 \\
\left(F_{M_{1}} \bullet F_{M_{2}}\right)((b, e)) & =\min \left\{F_{M_{1}}(b), F_{M_{2}}(e)\right\} \\
& =\min \{0.7,0.6\}=0.6
\end{aligned}
$$

for $b \in V_{1}$ and $e \in V_{2}$.

For the edge $(a, c)(b, d)$, we calculate the membership and non-membership values to be

$$
\begin{gathered}
\left(T_{N_{1}} \bullet T_{N_{2}}\right)((a, c)(b, d))=T_{N_{1}}(a b)=0.1, \\
\left(F_{N_{1}} \bullet F_{N_{2}}\right)((a, c)(b, d))=F_{N_{1}}(a b)=0.8
\end{gathered}
$$

for $a b \in E_{1}$ and $c \neq d$.

Similarly, we can find the membership and non-membership values for all the remaining vertices and edges.

Proposition 3.34. The residue product of two vague graphs $\mathbf{G}_{1}$ and $\mathbf{G}_{2}$ is a vague graph.

Proof: Let $\mathbf{G}_{1}=\left(M_{1}, N_{1}\right)$ and $\mathbf{G}_{2}=\left(M_{2}, N_{2}\right)$ be two vague graphs on crisp graphs $G_{1}=\left(V_{1}, E_{1}\right)$ and $G_{2}=\left(V_{2}, E_{2}\right)$, 
$a(0.1,0.5)$

FIGURE $17 \mid G_{1}$
$(0.1,0.8)$

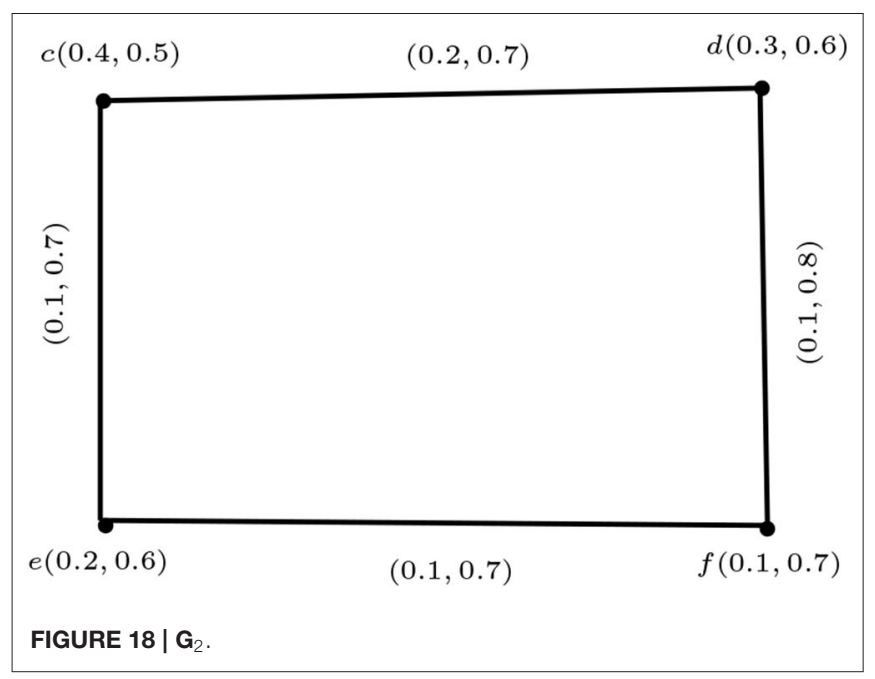

respectively, and let $\left(\left(m_{1}, m_{2}\right)\left(n_{1}, n_{2}\right)\right) \in E_{1} \times E_{2}$. If $m_{1} n_{1} \in E_{1}$ and $m_{2} \neq n_{2}$, then

$$
\begin{aligned}
& \left(T_{N_{1}} \bullet T_{N_{2}}\right)\left(\left(m_{1}, m_{2}\right)\left(n_{1}, n_{2}\right)\right)=T_{N_{1}}\left(m_{1} n_{1}\right) \\
& \leq \min \left\{T_{M_{1}}\left(m_{1}\right), T_{M_{1}}\left(n_{1}\right)\right\} \\
& \leq \max \left\{\min \left\{T_{M_{1}}\left(m_{1}\right), T_{M_{1}}\left(n_{1}\right)\right\}, \min \left\{T_{M_{2}}\left(m_{2}\right), T_{M_{2}}\left(n_{2}\right)\right\}\right\} \\
& =\min \left\{\max \left\{T_{M_{1}}\left(m_{1}\right), T_{M_{1}}\left(n_{1}\right)\right\}, \max \left\{T_{M_{2}}\left(m_{2}\right), T_{M_{2}}\left(n_{2}\right)\right\}\right\} \\
& =\min \left\{\left(T_{M_{1}} \bullet T_{M_{2}}\right)\left(m_{1}, m_{2}\right),\left(T_{M_{1}} \bullet T_{M_{2}}\right)\left(n_{1}, n_{2}\right)\right\}, \\
& \left(F_{N_{1}} \bullet F_{N_{2}}\right)\left(\left(m_{1}, m_{2}\right)\left(n_{1}, n_{2}\right)\right)=F_{N_{1}}\left(m_{1} n_{1}\right) \\
& \geq \max \left\{F_{M_{1}}\left(m_{1}\right), F_{M_{1}}\left(n_{1}\right)\right\} \\
& \geq \min \left\{\max \left\{F_{M_{1}}\left(m_{1}\right), F_{M_{1}}\left(n_{1}\right)\right\}, \max \left\{F_{M_{2}}\left(m_{2}\right), F_{M_{2}}\left(n_{2}\right)\right\}\right\} \\
& =\max \left\{\min \left\{F_{M_{1}}\left(m_{1}\right), F_{M_{1}}\left(n_{1}\right)\right\}, \min \left\{F_{M_{2}}\left(m_{2}\right), F_{M_{2}}\left(n_{2}\right)\right\}\right\} \\
& =\max \left\{\left(F_{M_{1}} \bullet F_{M_{2}}\right)\left(m_{1}, m_{2}\right),\left(F_{M_{1}} \bullet F_{M_{2}}\right)\left(n_{1}, n_{2}\right)\right\},
\end{aligned}
$$

which completes the proof.

Definition 3.35. Let $\mathbf{G}_{1}=\left(M_{1}, N_{1}\right)$ and $\mathbf{G}_{2}=\left(M_{2}, N_{2}\right)$ be two vague graphs. For any vertex $\left(m_{1}, m_{2}\right) \in V_{1} \times V_{2}$ we define

$$
\begin{aligned}
\left(d_{T}\right)_{\mathbf{G}_{1} \bullet \mathbf{G}_{2}}\left(m_{1}, m_{2}\right) & =\sum_{\left(m_{1}, m_{2}\right)\left(n_{1}, n_{2}\right) \in E_{1} \times E_{2}}\left(T_{N_{1}} \bullet T_{N_{2}}\right)\left(\left(m_{1}, m_{2}\right)\left(n_{1}, n_{2}\right)\right) \\
& =\sum_{m_{1} n_{1} \in E_{1}, m_{2} \neq n_{2}} T_{N_{1}}\left(m_{1} n_{1}\right) \\
& =\left(d_{T}\right)_{\mathbf{G}_{1}}\left(m_{1}\right),
\end{aligned}
$$

$$
\begin{aligned}
\left(d_{F}\right)_{\mathbf{G}_{1} \bullet \mathbf{G}_{2}}\left(m_{1}, m_{2}\right) & =\sum_{\left(m_{1}, m_{2}\right)\left(n_{1}, n_{2}\right) \in E_{1} \times E_{2}}\left(F_{N_{1}} \bullet F_{N_{2}}\right)\left(\left(m_{1}, m_{2}\right)\left(n_{1}, n_{2}\right)\right) \\
& =\sum_{m_{1} n_{1} \in E_{1}, m_{2} \neq n_{2}} F_{N_{1}}\left(m_{1} n_{1}\right) \\
& =\left(d_{F}\right)_{\mathbf{G}_{1}}\left(m_{1}\right) .
\end{aligned}
$$

Definition 3.36. Let $\mathbf{G}_{1}=\left(M_{1}, N_{1}\right)$ and $\mathbf{G}_{2}=\left(M_{2}, N_{2}\right)$ be two vague graphs. For any vertex $\left(m_{1}, m_{2}\right) \in V_{1} \times V_{2}$ we define

$$
\begin{aligned}
\left(t d_{T}\right)_{\mathbf{G}_{1} \bullet \mathbf{G}_{2}}\left(m_{1}, m_{2}\right)=\sum_{\left(m_{1}, m_{2}\right)}\left(T_{\left.n_{1}, n_{2}\right) \in E_{1} \times E_{2}} \bullet T_{N_{2}}\right)\left(\left(m_{1}, m_{2}\right)\left(n_{1}, n_{2}\right)\right) \\
+\left(T_{M_{1}} \bullet T_{M_{2}}\right)\left(m_{1}, m_{2}\right) \\
=\sum_{m_{1} n_{1} \in E_{1}, m_{2} \neq n_{2}} T_{N_{1}}\left(m_{1} n_{1}\right)+\min \left\{T_{M_{1}}\left(m_{1}\right), T_{M_{2}}\left(m_{2}\right)\right\} \\
=\sum_{m_{1} n_{1} \in E_{1}, m_{2} \neq n_{2}} T_{N_{1}}\left(m_{1} n_{1}\right)+T_{M_{1}}\left(m_{1}\right)+T_{M_{2}}\left(m_{2}\right) \\
\quad-\max \left\{T_{M_{1}}\left(m_{1}\right), T_{M_{2}}\left(m_{2}\right)\right\} \\
=\left(t d_{T}\right)_{\mathbf{G}_{1}}\left(m_{1}\right)+T_{M_{2}}\left(m_{2}\right)-\max \left\{T_{M_{1}}\left(m_{1}\right), T_{M_{2}}\left(m_{2}\right)\right\}
\end{aligned}
$$

$$
\begin{aligned}
& \left(t d_{F}\right)_{\mathbf{G}_{1} \bullet \mathbf{G}_{2}}\left(m_{1}, m_{2}\right)=\sum_{\left(m_{1}, m_{2}\right)}\left(F_{\left.n_{1}, n_{2}\right) \in E_{1} \times E_{2}} \bullet F_{N_{2}}\right)\left(\left(m_{1}, m_{2}\right)\left(n_{1}, n_{2}\right)\right) \\
& +\left(F_{M_{1}} \bullet F_{M_{2}}\right)\left(m_{1}, m_{2}\right) \\
& =\sum_{m_{1} n_{1} \in E_{1}, m_{2} \neq n_{2}} F_{N_{1}}\left(m_{1} n_{1}\right)+\max \left\{F_{M_{1}}\left(m_{1}\right), F_{M_{2}}\left(m_{2}\right)\right\} \\
& =\sum_{m_{1} n_{1} \in E_{1}, m_{2} \neq n_{2}} F_{N_{1}}\left(m_{1} n_{1}\right)+F_{M_{1}}\left(m_{1}\right)+F_{M_{2}}\left(m_{2}\right) \\
& \quad-\min \left\{F_{M_{1}}\left(m_{1}\right), F_{M_{2}}\left(m_{2}\right)\right\} \\
& =\left(t d_{F}\right)_{\mathbf{G}_{1}}\left(m_{1}\right)+F_{M_{2}}\left(m_{2}\right)-\min \left\{F_{M_{1}}\left(m_{1}\right), F_{M_{2}}\left(m_{2}\right)\right\} .
\end{aligned}
$$

Example 3.37. In this example we find the degree and total degree of the vertex $(b, e)$ in Example 3.33:

$$
\begin{aligned}
\left(d_{T}\right)_{\mathbf{G}_{1} \bullet \mathbf{G}_{2}}(b, e) & =\left(d_{T}\right)_{\mathbf{G}_{1}}(b) \\
& =0.1+0.1=0.2, \\
\left(d_{F}\right)_{\mathbf{G}_{1} \bullet \mathbf{G}_{2}}(b, e) & =\left(d_{F}\right)_{\mathbf{G}_{1}}(b) \\
& =0.8+0.8=1.6 .
\end{aligned}
$$

Therefore, 


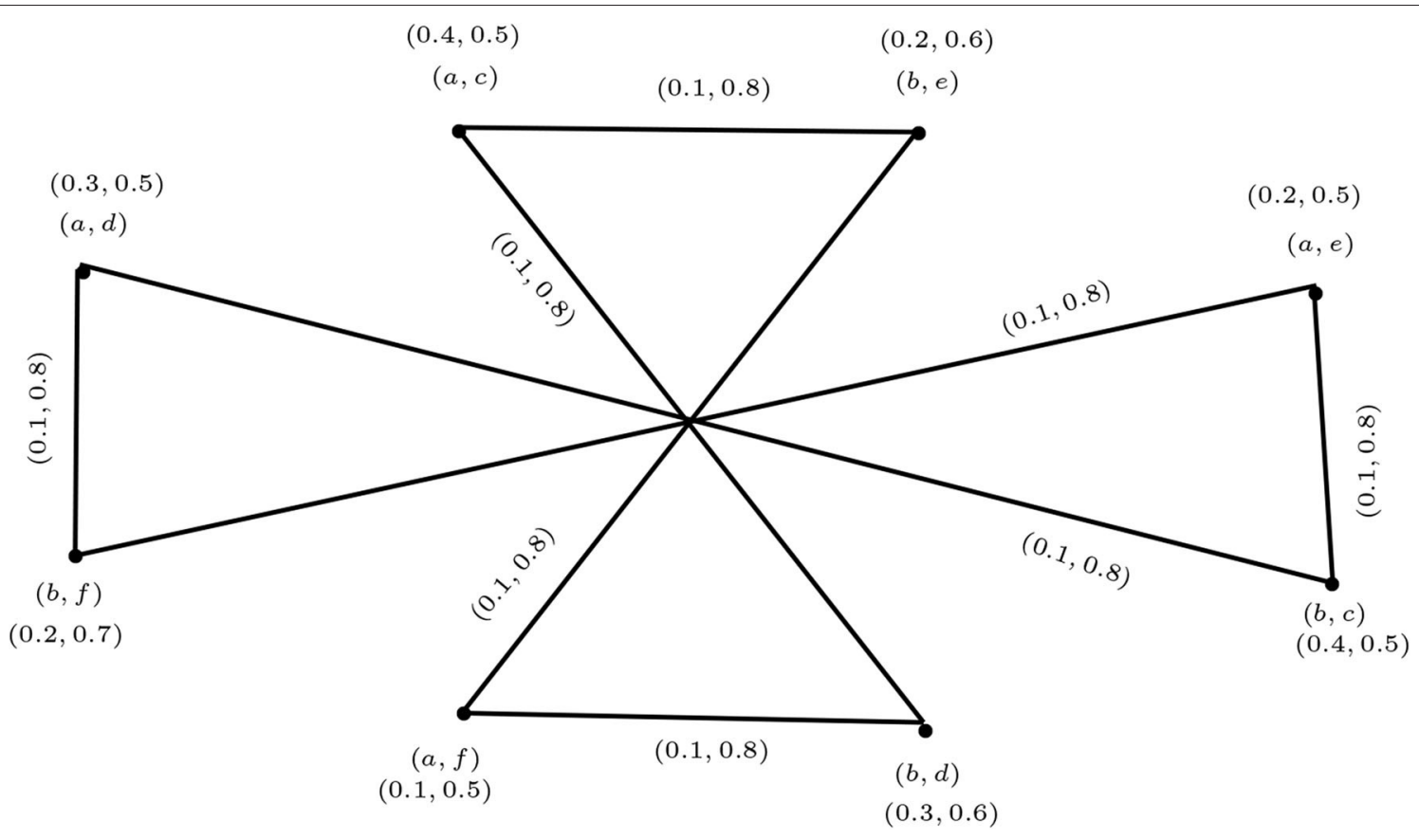

FIGURE $19 \mid G_{1} \bullet G_{2}$.

TABLE 1 | The vague relation $L(P \rightarrow S)$.

\begin{tabular}{lcccc}
\hline $\boldsymbol{L}$ & Heartburn & Coughing & Pain during swallowing & Weight loss \\
\hline Shahbaz & $(0.8,0)$ & $(0.6,0.2)$ & $(0.2,0.3)$ & $(0.1,0.6)$ \\
Faisal & $(0.3,0.1)$ & $(0.3,0.5)$ & $(0.3,0.6)$ & $(0.2,0.6)$ \\
Shoaib & $(0.5,0.2)$ & $(0.1,0.2)$ & $(0.1,0.8)$ & $(0.3,0.4)$ \\
Danish & $(0.4,0.4)$ & $(0,0.3)$ & $(0.5,0.4)$ & $(0.5,0.3)$
\end{tabular}

The total degree of $(b, e)$ is given by

$$
\begin{aligned}
\left(t d_{T}\right)_{\mathbf{G}_{1} \bullet \mathbf{G}_{2}}(b, e) & =\left(t d_{T}\right)_{\mathbf{G}_{1}}(b)+T_{M_{2}}(e)-\max \left\{T_{M_{1}}(b), T_{M_{2}}(e)\right\} \\
& =(0.2+0.2)+0.2-\max (0.2,0.2) \\
& =0.4 \\
\left(t d_{F}\right)_{\mathbf{G}_{1} \bullet \mathbf{G}_{2}}(b, e) & =\left(t d_{F}\right)_{\mathbf{G}_{1}}(b)+F_{M_{2}}(e)-\min \left\{F_{M_{1}}(b), F_{M_{2}}(e)\right\} \\
& =(0.8+0.8)+0.6-\min (0.7,0.6) \\
& =1.6 .
\end{aligned}
$$

Therefore,

$$
(t d)_{\mathbf{G}_{1} \bullet \mathbf{G}_{2}}(b, e)=(0.4,1.6) .
$$

Similarly, we can find the degree and total degree of all vertices in $\mathbf{G}_{1} \bullet \mathbf{G}_{2}$.
TABLE 2 | The vague relation $R(S \rightarrow D)$.

\begin{tabular}{lcccc}
\hline $\boldsymbol{R}$ & $\begin{array}{c}\text { Cancer of } \\
\text { kidney }\end{array}$ & $\begin{array}{c}\text { Cancer of } \\
\text { colon }\end{array}$ & $\begin{array}{c}\text { Cancer of } \\
\text { breast }\end{array}$ & $\begin{array}{c}\text { Cancer of } \\
\text { bladder }\end{array}$ \\
\hline Heartburn & $(0.1,0.3)$ & $(0.6,0.1)$ & $(0.2,0.3)$ & $(0.7,0.1)$ \\
Coughing & $(0.5,0.4)$ & $(0.3,0.4)$ & $(0.3,0.5)$ & $(0.2,0.6)$ \\
Pain during & $(0.3,0.6)$ & $(0.5,0.2)$ & $(0.6,0.1)$ & $(0.7,0.1)$ \\
swallowing & & & & $(0.7,0.1)$ \\
Weight loss & $(0.2,0.3)$ & $(0.6,0.3)$ & $(0.5,0.2)$ & \\
\hline
\end{tabular}

\section{APPLICATION OF VAGUE SETS TO MEDICAL DIAGNOSIS}

Following the approach outlined by De et al. [18], we will apply vague sets to medical diagnosis by using a max-min-max composition in terms of vague relations. First, we use vague sets to define the disease symptoms. Then, we describe medical knowledge in terms of vague relations. Finally, we determine a diagnosis on the basis of vague relations. Consider four patients named Shahbaz, Shoaib, Faisal, and Danish, and define the set of patients $P=$ \{Shahbaz, Shoaib, Faisal, Danish\}. Let the set of symptoms under consideration be $S=$ \{heartburn, coughing, pain during swallowing, weight loss\}. A vague relation $L$ is available from set $P$ to set $S$, and this is summarized in Table $\mathbf{1}$.

Cancer is a group of dangerous and prevalent diseases, and represents one of humankind's greatest medical challenges. Many people are diagnosed with late-stage cancer that is difficult or 
TABLE 3 | The composition $M(P \rightarrow D)$ of vague relations $L$ and $R$.

\begin{tabular}{lcccc}
\hline $\boldsymbol{M}$ & $\begin{array}{c}\text { Cancer of } \\
\text { kidney }\end{array}$ & $\begin{array}{c}\text { Cancer of } \\
\text { colon }\end{array}$ & $\begin{array}{c}\text { Cancer of } \\
\text { breast }\end{array}$ & $\begin{array}{c}\text { Cancer of } \\
\text { bladder }\end{array}$ \\
\hline Shahbaz & $(0.5,0.3)$ & $(0.6,0.1)$ & $(0.2,0.3)$ & $(0.7,0.1)$ \\
Faisal & $(0.3,0.3)$ & $(0.3,0.1)$ & $(0.3,0.3)$ & $(0.3,0.1)$ \\
Shoaib & $(0.2,0.3)$ & $(0.5,0.2)$ & $(0.3,0.3)$ & $(0.5,0.2)$ \\
Danish & $(0.5,0.3)$ & $(0.5,0.3)$ & $(0.5,0.3)$ & $(0.5,0.3)$ \\
\hline
\end{tabular}

TABLE $4 \mid S_{R}$, the best version of $R$ determined by the formula $S_{R}=T_{R}-F_{R} \pi_{R}$.

\begin{tabular}{lcccc}
\hline $\boldsymbol{S}_{\boldsymbol{R}}$ & $\begin{array}{c}\text { Cancer of } \\
\text { kidney }\end{array}$ & $\begin{array}{c}\text { Cancer of } \\
\text { colon }\end{array}$ & $\begin{array}{c}\text { Cancer of } \\
\text { breast }\end{array}$ & $\begin{array}{c}\text { Cancer of } \\
\text { bladder }\end{array}$ \\
\hline Shahbaz & 0.44 & 0.57 & 0.05 & 0.68 \\
Faisal & 0.18 & 0.24 & 0.18 & 0.24 \\
Shoaib & 0.05 & 0.44 & 0.18 & 0.44 \\
Danish & 0.44 & 0.44 & 0.44 & 0.44 \\
\hline
\end{tabular}

impossible to treat because of a lack of awareness of the disease symptoms. Mathematical models involving vague sets can be used to determine the most likely diagnosis given a set of symptoms that a patient presents with.

There are many different types of cancers; here we focus on a few of the more life-threatening kinds: (1) kidney cancer, (2) colon cancer, (3) breast cancer, and (4) bladder cancer. We define the set of diagnoses to be $D=$ \{cancer of kidney, cancer of colon, cancer of breast, cancer of bladder\}. The vague relation $R(S \rightarrow D)$ from the set of symptoms to the set of diagnoses is given in Table 2. The composition $M(P \rightarrow D)$ of the vague relations $L$ and $R$ is shown in Table 3; it gives the diagnosis for each patient via the formulas

$$
\begin{aligned}
& T_{M}\left(p_{i}, d_{k}\right)=\bigvee_{s \in S}\left[T_{L}\left(p_{i}, s\right) \wedge T_{R}\left(s, d_{k}\right)\right], \\
& F_{M}\left(p_{i}, d_{k}\right)=\bigwedge_{s \in S}\left[F_{L}\left(p_{i}, s\right) \vee F_{R}\left(s, d_{k}\right)\right],
\end{aligned}
$$

where $p_{i}$ denotes the patients, $d_{k}$ denotes the different diagnoses, $\wedge=\min$, and $\vee=\max$.

\section{REFERENCES}

1. Zadeh L. Fuzzy sets. Inform Control. (1965) 8:338-53.

2. Rosenfeld A. Fuzzy graphs. In: Zadeh LA, Fu KS, Shimura M, editors. Fuzzy Sets and Their Application. New York, NY: Academic Press (2006). p. 77-95.

3. Bhattacharya P. Some remarks on fuzzy graphs. Pattern Recogn Lett. (1987) 6:297-302.

4. Bhattacharya P, Suraweera F. An Algorithm to compute the max-min powers and a property of fuzzy graphs. Pattern Recogn Lett. (1991) 12:413-20.

5. Nagoor Gani A, Latha SR. On irregular fuzzy graphs. Appl Math Sci. (2012) 6:517-23.

6. Nagoor Gani A, Basheer Ahamed M. Order and size in fuzzy graphs. Bull Pure Appl Sci. (2003) 22:145-8.

7. Gau WL, Buehrer DJ. Vague sets. IEEE Trans Syst Man Cybernet. (1993) 23:610-4.
Shown in Table 4 is $S_{R}$, the best version of diagnosis for this set of patients, which is determined by the formula $S_{R}=T_{R}-F_{R} \pi_{R}$. It is very important because the max-min-max rule alone fails to provide exact information.

\section{CONCLUSION}

Compared with fuzzy models, vague models offer greater compatibility and flexibility. A vague graph is a type of extension of a fuzzy graph, and is used widely in the field of computer science. We have defined four new operations of a vague graph, called the maximal product, rejection, symmetric difference, and residue product. We have discussed their properties and provided examples on finding the degree of a vertex and the total degree of vertices of graphs that meet specific conditions. We have formulated and proved theorems for these graphs by using the concept of degree of a vertex and total degree of a vertex of a graph. Furthermore, we have presented an application of vague sets to the medical diagnosis of four types of cancer. In future work we will explore further properties relating to vague graphs and bipolar vague graphs.

\section{DATA AVAILABILITY STATEMENT}

All datasets generated for this study are included in the article/supplementary material.

\section{AUTHOR CONTRIBUTIONS}

All authors listed have made a substantial, direct and intellectual contribution to the work, and approved it for publication.

\section{FUNDING}

This work was supported by the National Key R\&D Program of China (grant no. 2019YFA0706402), the Natural Science Foundation of Guangdong Province (grant no. 2018A0303130115), and the Guangzhou Academician and Expert Workstation (no. 20200115-9).
8. Ramakrishna N. Vague graphs. Int J Comput Cogn. (2009) 7:51-8.

9. Borzooei RA, Rashmanlou H. New concepts of vague graphs. Int J Mach Learn Cybernet. (2015) 8:1081-92. doi: 10.1007/s13042-0150475-x

10. Rashmanlou H, Borzooei RA. Product vague graphs and its applications. $J$ Intell Fuzzy Syst. (2016) 30:371-82. doi: 10.3233/IFS-151762

11. Boorzooei RA, Rashmanlou H, Samanta S, Pal M. Regularity of vague graphs. J Intell Fuzzy Syst. (2016) 30:3681-9. doi: 10.3233/IFS-162114

12. Borzooei RA, Rashmanlou H, Samanta S, Pal M. New concepts of vague competition graphs. J Intell Fuzzy Syst. (2016) 31:69-75. doi: 10.3233/IFS-162121

13. Kumar K, Lavanya S, Broumi S, Rashmanlou H. New concepts of coloring in vague graphs with application. J Intell Fuzzy Syst. (2017) 33:1715-21. doi: 10.3233 /JIFS-17489

14. Atanassov K. Intuitionistic fuzzy sets. Fuzzy Sets Syst. (1986) 20:87-96. 
15. Parvathi R, Karunambigai MG. Intuitionistic fuzzy graphs, computational intelligence, theory and applications. In: International Conference in Germany. Berlin (2006). p. 18-20.

16. Devi M, Ameenal Bibi K, Rashmanlou H. New concepts in intuitionistic fuzzy labeling graphs. Int $J$ Adv Intell Paradigms. (2020).

17. Berge C. Graphs and Hyper Graphs. Amsterdam: North-Holland Publishing Company (1973).

18. De SK, Biswas R, Roy AR. An application of intuitionistic fuzzy sets in medical diagnosis. Fuzzy Sets Syst. (2001) 117:209-13. doi: 10.1016/S0165-0114(98) 00235-8
Conflict of Interest: The authors declare that the research was conducted in the absence of any commercial or financial relationships that could be construed as a potential conflict of interest.

Copyright $\odot 2020$ Shao, Kosari, Shoaib and Rashmanlou. This is an open-access article distributed under the terms of the Creative Commons Attribution License (CC $B Y)$. The use, distribution or reproduction in other forums is permitted, provided the original author(s) and the copyright owner(s) are credited and that the original publication in this journal is cited, in accordance with accepted academic practice. No use, distribution or reproduction is permitted which does not comply with these terms. 